\title{
Parameters And State Estimates Of Sex Based Covid-19 Model Using Kenya Data, Nonlinear Least Square And Interpolating Polynomials
}

\author{
Cyrus Gitonga Ngari $\square$, Dominic Makaa Kitavi $\square$, Paul Muriithi Ngari $\square$ \& David Mugo Muchangi $\square$ \\ Department of Mathematics, Computing, and Information Technology; University of Embu \\ Disease Surveillance and Response Unit; Embu Teaching and Referral Hospital \\ DOI: 10.29322/IJSRP.11.05.2021.p11348 \\ http://dx.doi.org/10.29322/IJSRP.11.05.2021.p11348
}

\begin{abstract}
COVID-19 spread in Kenya has been growing at a very high rate in the recent past. According to the Kenya's ministry of health, the confirmed COVID-19 infections as of $19^{\text {th }}$ July 2020 was 13,353 with recorded 5,122 recoveries and 234 deaths. Based on quarantine data, there is media speculation about COVID-19 manifesting gender dimension, however, no studies have been carried out to establish the gender-based dimension in the community. This paper aimed at: formulating gender based Mathematical model, estimate gender-based disease burden in the community using quarantine data and using estimated parameters and states to predict dynamics of the disease in the quarantine centers. Mathematical compartment model was developed using characteristic and status of disease. Daily number of infectious and exposed in the community was estimated using interpolating polynomials. Nonlinear least square was used to fit observed data in the developed model. Prediction of the initial value problem was carried out using MATLAB inbuilt ode solver. Daily estimate of states in Figures 8 and 9 confirms that COVID-19 is also burdening more males in the community than females. Simulation using MATLAB indicated that the number of individuals who will remain constantly infected after disease induced deaths and recoveries ranges between $(567-219)$ and $(363-116)$ for males and females respectively. Future studies should focus on Mathematical model analysis and predictions of disease burden in the community.
\end{abstract}

Index Terms- COVID-19, Quarantine data, nonlinear least square, interpolating polynomials, Predictions.

\section{INTRODUCTION}

$I_{n}^{n}$ late December 2019, there occurred an outbreak of a pneumonia-like illness in the Hubei province of Wuhan, China. The disease was caused by a novel coronavirus and spread rapidly throughout China and across the world. The disease, which was later named officially by World Health Organization (WHO) as COVID-19 (Corona Virus Disease, 2019), had in the early days of detection been referred to as "2019 novel coronavirus" abbreviated as 2019-nCoV, and subsequently referred to as "severe acute respiratory syndrome coronavirus 2" abbreviated as SARS-CoV-2 (Zheng, Ma, Zhang, \& Xie, 2020) (Velavan \& Meyer, 2020) (Cascella, Rajnik, Cuomo, Dulebohn, \& Napoli,
2020). COVID-19 is reported to have been initially transmitted from animals to humans on Huanan seafood market in Wuhan (Velavan \& Meyer, 2020).

Since the first infections of COVID-19 were directly linked to Huanan Seafood Wholesale Market's exposure, animal-tohuman transmission of the virus was presumed to be the main mechanism. However, subsequent cases were not linked to this transmission mechanism but rather, human-to-human transmission of the virus was happening, and at a very high rate (Cascella, Rajnik, Cuomo, Dulebohn, \& Napoli, 2020) (U.S. Department of Labor, 2020) (Abiad, Arao, \& Dagli, 2020). The transmission occurs through respiratory droplets from coughing, sneezing, or talking. People can also get COVID-19 by touching surfaces or objects that have the COVID-19 causing virus on them and then touching their mouths and/or nose, or even possibly touching their eyes (U.S. Department of Labor, 2020) (Centers for Disease Control and Prevention and Others, 2020).

According to WHO, viral diseases continue to emerge and pose a great challenge in the healthcare system. Since 2000, several viral epidemics such as the severe acute respiratory syndrome coronavirus (SARS-CoV) reported in 2002, H1N1 influenza reported in 2009, the Middle East respiratory syndrome coronavirus (MERS-CoV) reported in 2012, have been recorded (Cascella, Rajnik, Cuomo, Dulebohn, \& Napoli, 2020).

As of $19^{\text {th }}$ July 2020, confirmed COVID-19 infections across the world was 14,640,244 with recorded 8,729,761 recoveries and 612,377 deaths. In Kenya, the confirmed COVID19 infections were 13,353 with recorded 5,122 recoveries and 234 deaths (Worldometer, 2020). The world's recovery and death rates as of $19^{\text {th }}$ July 2020 were approximately $59.6 \%$ and $4.2 \%$ respectively, as compared to Kenya's recovery and death rates of $38.4 \%$ and $1.8 \%$ respectively, as of $19^{\text {th }}$ July 2020 . The increased number of confirmed COVID-19 cases, continuing daily deaths, fear, stigma, cost of medication, slow economic growth, among others, makes prevention and control to be extremely important. Given the fragile health system in Kenya, COVID-19 pandemic can potentially paralyze the health system at the expense of primary healthcare requirements. In fact, some healthcare centers such as Pumwani Maternity hospital in Nairobi have already been temporarily closed.

According to the currently available information, COVID19 mainly invades alveolar epithelial cells which results into respiratory symptoms. The symptoms are known to be more 
severe in patients with cardiovascular diseases (CVDs) hence increased risk of death to such patients. Thus, having an understanding of the damage caused by COVID-19 to the cardiovascular system is of great importance to enable treatment of the patients to be timely and effective in order to reduce the mortality rate (Zheng, Ma, Zhang, \& Xie, 2020).

There are symptomatic (showing symptoms of illness) and asymptomatic (showing no symptoms) patients. The proportion of COVID-19 infected individuals who remain asymptomatic is yet to be established. Clinical manifestations of COVID-19 in symptomatic patients usually start within 7 days of infection. The symptoms include fever, cough, nasal congestion, sore throat, fatigue, headache, dyspnea (difficulty in breathing), loss of taste and/or smell, diarrhea, and vomiting.

Usually, Pneumonia would occur within 14 to 21 days of a COVID-19 symptomatic infection (Velavan \& Meyer, 2020) (Cascella, Rajnik, Cuomo, Dulebohn, \& Napoli, 2020) (U.S. Department of Labor, 2020).

This study relies heavily on interpolating polynomials and nonlinear least square methods to fit the observed data in the developed model. Prime purpose of the two methods is to approximate nonlinear data model with a linear model to be able to estimate some unknown parameters of interest. To avoid distraction from the current focus on the study of gender-based COVID-19 infection in Kenya, detailed discussion on interpolating polynomials and nonlinear least square methods is hereby avoided. However, interested readers are directed to (Philips, 2003) (Robin, 1972) (Zippel, 1990) (Erdos \& Turan, 1940), among others, for more information on interpolating polynomials and (Jr, Gay, \& Walsh, 1981) (Wu, 1981) (Golub \&
Pereyra, 2003) (Gill \& Murray, 1978) (Johnson \& Frasier, 1985), among others, for more information on nonlinear least squares (NLS).

\section{METHODS}

We used population based compartmental model depending on status of infection and sex. We considered a closed community where the vital dynamics of births, immigration, emigration, and natural death were assumed to be not significant.

The total population $\mathrm{N}(\mathrm{t})$ was classified into two categories based on sex, $N_{M}(t)$ being total population of males and $N_{F}(t)$ being total population of females. Males were categorized as $\mathrm{S}_{\mathrm{M}}(t)$ being susceptible males, $L_{M}(t)$ being males exposed to Covid-19 but in latency state, $I_{M}(t)$ being COVID-19 infectious males in community and $\mathrm{Q}_{\mathrm{M}}(t)$ being either infectious or exposed males in quarantine centers. Females were categorized as $\mathrm{S}_{\mathrm{F}}(t)$ being susceptible females, $L_{F}(t)$ being females exposed to Covid-19 but in latency state, $I_{F}(t)$ being COVID-19 infectious Females in community and $\mathrm{Q}_{\mathrm{F}}(t)$ being either infectious or exposed females in quarantine centers. $\square$ is the infection rate, $\square_{1}$ and $\square 2$ are COVID-19 recovery rates for $\mathrm{Q}_{\mathrm{M}}$ and $\mathrm{Q}_{\mathrm{F}}$ respectively, $\square_{1}$ and $\square 2$ are COVID-19 progression rates from $L_{M}$ and $L_{F}$ to $I_{M}$ and $I_{F}$ respectively, $\square$ 1and $\square \mathbf{2}$ are COVID-19 enlisting rates to $\mathrm{Q}_{\mathrm{M}}$ and $\mathrm{Q}_{\mathrm{F}}$ respectively, $\square$ M1 and $\square$ F1 are COVID-19 induced death rates for $\mathrm{I}_{\mathrm{M}}$ and $\mathrm{I}_{\mathrm{F}}$ respectively and $\square \mathbf{M} 2$ and $\square$ F2 are COVID-19 induced death rates for $\mathrm{Q}_{\mathrm{M}}$ and $\mathrm{Q}_{\mathrm{F}}$ respectively.

\section{Model Equations:}

$$
\begin{aligned}
& \frac{d S_{M}}{d t}=\boldsymbol{\theta}_{1} Q_{M}-\left(\mathbf{1}-\boldsymbol{\varepsilon}_{M}\right) \lambda S_{M} \\
& \frac{d L_{M}}{d t}=\left(\mathbf{1}-\varepsilon_{M}\right) \lambda S_{M}-\left(\omega_{1}+\boldsymbol{h}_{\boldsymbol{m}} \gamma_{\mathbf{1}}\right) L_{M} \\
& \frac{d I_{M}}{d t}=\omega_{1} L_{M}-\left(\left(\mathbf{1}-\boldsymbol{h}_{\boldsymbol{m}}\right) \gamma_{\mathbf{1}}+\delta_{\mathbf{M} \mathbf{1}}\right) I_{M} \\
& \frac{d Q_{M}}{d t}=\left(\mathbf{1}-\boldsymbol{h}_{\boldsymbol{m}}\right) \gamma_{\mathbf{1}} I_{M}+\boldsymbol{h}_{\boldsymbol{m}} \gamma_{\mathbf{1}} I_{M}-\left(\boldsymbol{\theta}_{\mathbf{1}}+\boldsymbol{\delta}_{\mathrm{M} 2}\right) Q_{M} \\
& \frac{d S_{F}}{d t}=\theta_{2} Q_{F}-\left(\mathbf{1}-\varepsilon_{F}\right) \lambda S_{F} \\
& \frac{d L_{F}}{d t}=\left(\mathbf{1}-\boldsymbol{\varepsilon}_{F}\right) \lambda S_{F}-\left(\omega_{2}+\boldsymbol{h}_{\boldsymbol{f}} \gamma_{2}\right) L_{F} \\
& \frac{d I_{F}}{d t}=\omega_{2} L_{F}-\left(\left(\mathbf{1}-\boldsymbol{h}_{\boldsymbol{f}}\right) \gamma_{\mathbf{2}}+\boldsymbol{\delta}_{\mathbf{F} \mathbf{1}}\right) I_{F} \\
& \frac{d Q_{F}}{d t}=\left(\mathbf{1}-\boldsymbol{h}_{\boldsymbol{f}}\right) \gamma_{2} I_{F}+\boldsymbol{h}_{\boldsymbol{f}} \gamma_{2} I_{F}-\left(\boldsymbol{\theta}_{\mathbf{2}}+\boldsymbol{\delta}_{\mathrm{F} 2}\right) Q_{F} \\
& =\boldsymbol{\beta}\left(\frac{\mathrm{L}_{\mathrm{M}}+\eta_{1} \mathrm{I}_{\mathrm{M}}}{N_{M}}+\frac{\eta_{2} \mathrm{~L}_{\mathrm{F}}+\eta_{3} \mathrm{I}_{\mathrm{F}}}{N_{F}}\right) \\
& \mathbf{0} \leq \boldsymbol{h}_{\boldsymbol{m}}, \boldsymbol{h}_{\boldsymbol{f}}, \square_{1}, \square_{2}, \square_{3} \leq \mathbf{1}, \\
& N_{M}+N_{F}=N \\
& S_{M}+L_{M}+I_{M}+Q_{M}=\boldsymbol{\Psi} \boldsymbol{N} ; S_{F}+L_{F}+I_{F}+Q_{F}=(1-\boldsymbol{\Psi})
\end{aligned}
$$




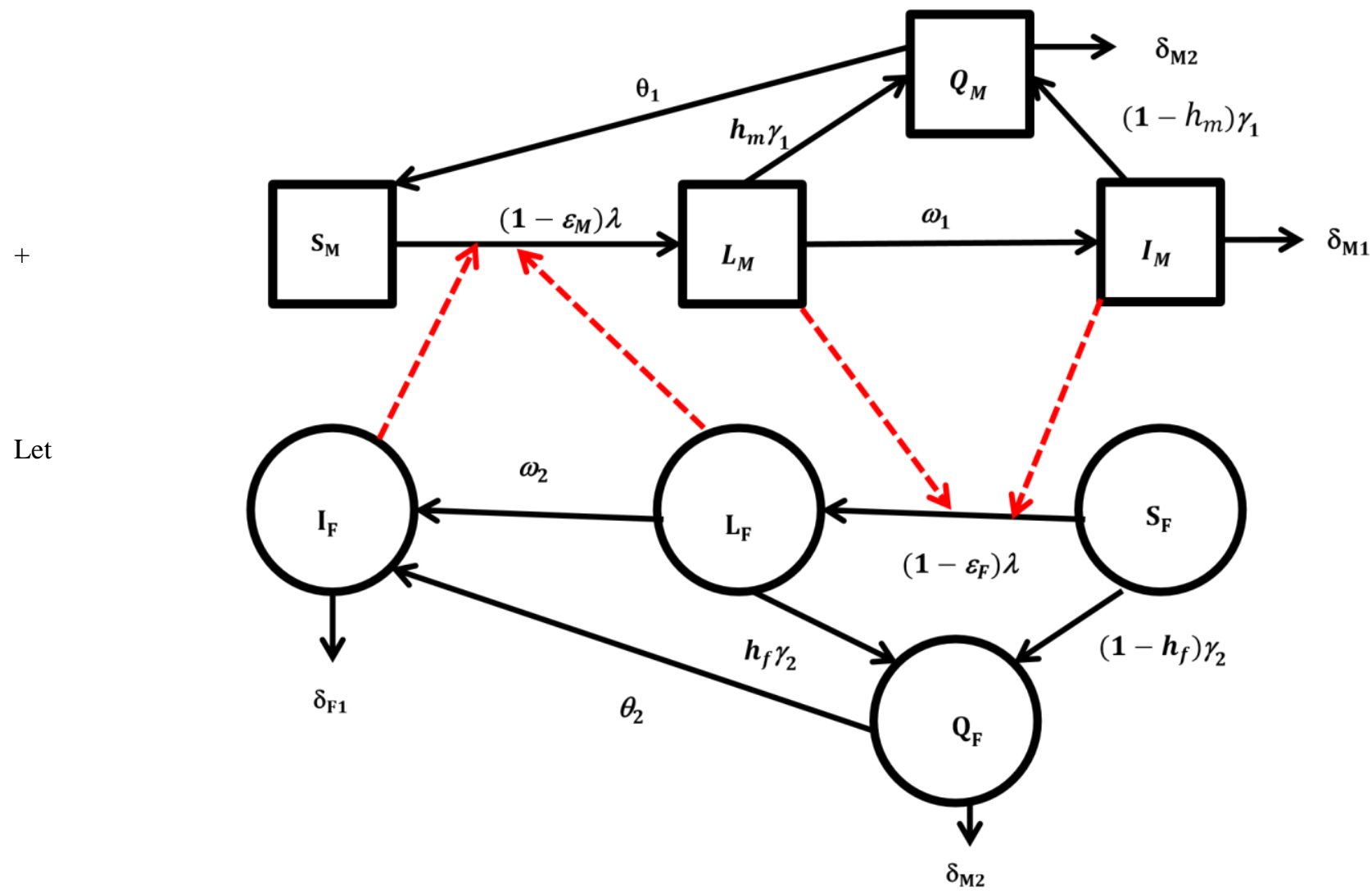

$s_{m}=\frac{S_{M}}{N_{M}} ; \mathrm{l}_{\mathrm{m}}=\frac{\mathrm{L}_{\mathrm{M}}}{N_{M}} ; \mathrm{i}_{\mathrm{m}}=\frac{\mathrm{I}_{\mathrm{M}}}{N_{M}} ; q_{m}=\frac{Q_{M}}{N_{M}} ; \mathrm{l}_{f}=\frac{\mathrm{L}_{\mathrm{F}}}{N_{F}} ; \mathrm{i}_{\mathrm{f}}=\frac{\mathrm{I}_{\mathrm{F}}}{N_{F}} ; q_{f}=\frac{Q_{F}}{N_{F}} ; s_{m}=\frac{S_{M}}{N_{M}} ; t=t ; \lambda=$

$\square=\square\left(l_{m}+\square_{1} i_{m}+\square_{2} l_{f}+\square{ }_{3} i_{f}\right)$

$\mathrm{s}_{\mathrm{m}}=\Psi-\mathrm{l}_{\mathrm{m}}-i_{m}-\mathrm{q}_{\mathrm{m}} ; s_{f}=1-\Psi-l_{f}-i_{f}-q_{f}$

$\frac{d l_{m}}{d t}=\left(1-\varepsilon_{M}\right) \beta\left(l_{m}+\eta_{1} i_{m}+\eta_{2} l_{f}+\eta_{3} i_{f}\right)\left(\Psi-l_{m}-i_{m}-q_{m}\right)-\left(\omega_{1}+h_{m} \gamma_{1}\right) l_{\mathrm{m}}$

$\frac{d i_{m}}{d t}=\omega_{1} l_{m}-\left(\left(1-h_{m}\right) \gamma_{1}+\delta_{\mathrm{M} 1}\right) i_{m}$

$\frac{d q_{m}}{d t}=\left(1-h_{m}\right) \gamma_{1} i_{m}+h_{m} \gamma_{1} l_{m}-\left(\theta_{1}+\delta_{\mathrm{M} 2}\right) \mathrm{q}_{\mathrm{m}}$

$\frac{d l_{f}}{d t}=\left(1-\varepsilon_{F}\right) \beta\left(l_{m}+\eta_{1} i_{m}+\eta_{2} l_{f}+\eta_{3} i_{f}\right)\left(1-\Psi-l_{f}-i_{f}-q_{f}\right)-\left(\omega_{2}+\mathrm{h} \gamma_{2}\right) l_{f}$

$\frac{d i_{f}}{d t}=\omega_{2} l_{f}-\left((1-\mathrm{h}) \gamma_{2}+\delta_{\mathrm{F} 1}\right) i_{f}$

$\frac{d q_{f}}{d t}=\left(1-h_{f}\right) \gamma_{2} i_{f}+h_{f} \gamma_{2} l_{f}-\left(\theta_{2}+\delta_{\mathrm{F} 2}\right) q_{f}$

The initial conditions of the model were:

$\mathrm{l}_{\mathrm{m}}(0) \geq 0 ; i_{m}(0) \geq 0 ; q_{m}(0) \geq 0 ; l_{f}(0) \geq 0 ; i_{f}(0) \geq 0 ; q_{f}(0) \geq 0$

Feasible region of the Quarantine equations

Theorem

The quarantine equations for males and Females lie in the positive region of the real domain Proof

Consider equation $\frac{\mathrm{d} \mathrm{q}_{\mathrm{m}}}{\mathrm{dt}}=\left(1-h_{m}\right) \gamma_{1} \mathrm{i}_{\mathrm{m}}+h_{m} \gamma_{1} \mathrm{l}_{\mathrm{m}}-\left(\theta_{1}+\delta_{\mathrm{M} 2}\right) \mathrm{q}_{\mathrm{m}}$. Clearly the term

$\left(1-h_{m}\right) \square_{1} \mathrm{i}_{\mathrm{m}}+h_{m} \square_{1} \mathrm{l}_{\mathrm{m}} \geq 0$. Ignoring the positive term, we obtain $\frac{d q_{m}}{d t} \geq-\left(\theta_{1}+\delta_{M 2}\right) q_{m}$.

On integration $(t) \geq q(0) e^{-\left(\square 1+\square M^{2}\right)} \geq 0$. Similarly, $(t) \geq q(0) e^{-\left(\square 2+\square F^{2}\right)} \geq 0$. Hence the theorem is proved. 


\section{Ministry of Health data from 18/4/2020 to 19/7/2020 (Table 3)}

Our study assumed that any case that Government is aware of is considered to be in quarantine. Therefore, all the data from Ministry of Health is a quarantine data.

The recovery rates and death rates were obtained and calculated using data from ministry of Health in table 3 as follows:

Death rate $=\frac{\text { Total Deaths }}{\text { Total infected }} ;$ Recovery rate $=\frac{\text { Total Recoveries }}{\text { Total infected }}$

Date 18/4/2020 was considered as initial condition of the data (at $t=0$ ), that is, Day zero and Date 19/7/2020 was considered as last date of the data (at $t=89$ ), that is, Day 89. Some days were omitted in Appendix 1 due to insufficiency of sex-based data. Using raw data from ministry of health, we obtained the following graphical presentations.

\section{Figure 1: Males and Females in Quarantine Figure 2: Consolidated Death Rates}

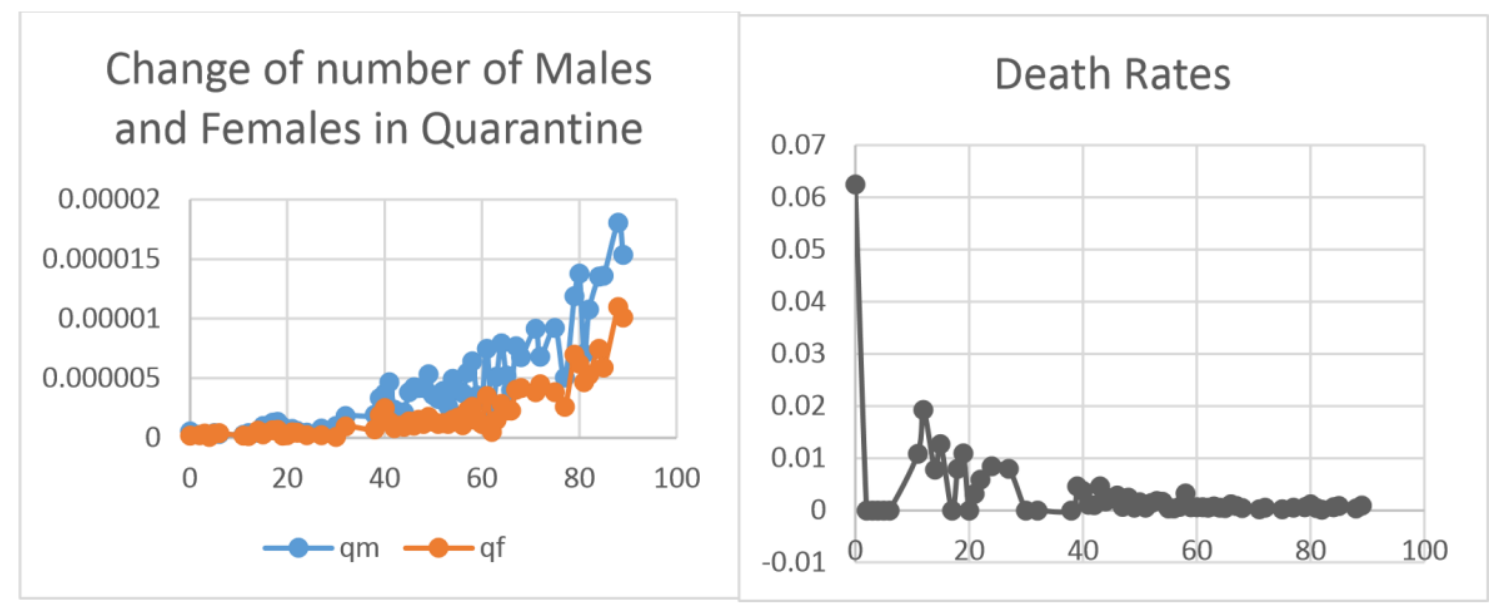

Figure 3: Consolidated Recovery Rates

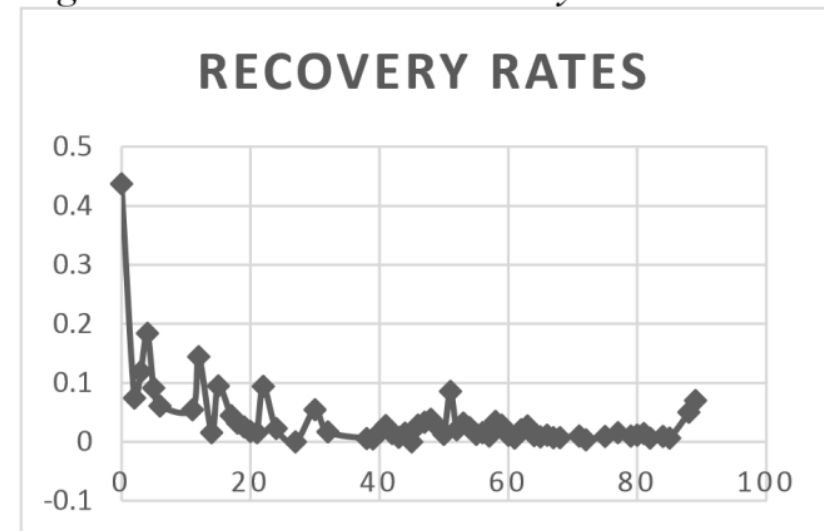

The descriptive results from figure 1 indicates that numbers of Males in quarantine is relatively higher than the number of females and it is continuing to rise with time. The consolidated maximum recovery rates and death rates for the 89 days are 0.4375 and 0.0625 , respectively. The consolidated minimum recovery rates and death rates for the 89 days are both zero. The consolidated average recovery rates and death rates for the 89 days are 0.038204375 and 0.003294081 , respectively.

\section{Parametrization of Model}

Consider males in Quarantine:

$\frac{d q_{m}}{d t}=\left(\mathbf{1}-\boldsymbol{h}_{\boldsymbol{m}}\right) \gamma_{\mathbf{1}} \boldsymbol{i}_{\boldsymbol{m}}+h_{m} \gamma_{\mathbf{1}} \boldsymbol{l}_{\boldsymbol{m}}-\left(\boldsymbol{\theta}_{\mathbf{1}}+\boldsymbol{\delta}_{\mathrm{M} 2}\right) \mathrm{q}_{\mathrm{m}}$ then

$\frac{d}{d t}\left(\ln \frac{q_{m}(t)}{q_{m}(0)}\right)=\left(\mathbf{1}-h_{m}\right) \gamma_{\mathbf{1}} \frac{\boldsymbol{i}_{\boldsymbol{m}}}{\boldsymbol{q}_{\boldsymbol{m}}(\boldsymbol{t})}+h_{m} \gamma_{\mathbf{1}} \frac{\boldsymbol{l}_{\boldsymbol{m}}}{q_{m}(t)}-\left(\boldsymbol{\theta}_{\mathbf{1}}+\boldsymbol{\delta}_{\mathbf{M} \mathbf{2}}\right)$

Fitting data of $(t)$ from figure 1 to a polynomial using MATLAB software, we obtain 
$\ln \frac{q_{m}(t)}{q_{m}(0)} \cong 3.8 \times 10^{-7} t^{4}-6.6 \times 10^{-5} t^{3}+0.0035 \times t^{2}-0.0077 t-0.48$

4th degree: norm of residuals $=2.8892$

Then

$\frac{d}{d t}\left(\ln \frac{q_{m}(t)}{q_{m}(0)}\right) \cong 4 \times 3.8 \times 10^{-7} t^{3}-3 \times 6.6 \times 10^{-5} t^{2}+2 \times 0.0035 t-0.0077$

Consider Females in Quarantine:

$\frac{d q_{f}}{d t}=\left(1-h_{f}\right) \gamma_{2} i_{f}+h_{f} \gamma_{2} l_{f}-\left(\theta_{2}+\delta_{\mathrm{F} 2}\right) q_{f}$, then

$\frac{d}{d t}\left(\ln \frac{q_{f}(t)}{q_{f}(0)}\right)=\left(1-h_{f}\right) \gamma_{2} \frac{i_{f}(t)}{q_{f}(t)}+h_{f} \gamma_{2} \frac{l_{f}(t)}{q_{f}(t)}-\left(\theta_{2}+\delta_{\mathrm{F} 2}\right)$

Fitting data of $(t)$ from figure 1 to a polynomial using MATLAB software, we obtain

$\ln \frac{q_{f}(t)}{q_{f}(0)} \cong 2.4 \times 10^{-7} t^{4}-4.3 \times 10^{-5} t^{3}+0.0026 t^{2}-0.017 t+0.27$

4th degree: norm of residuals $=4.0487$. Then

$\frac{d}{d t}\left(\ln \frac{q_{m}(t)}{q_{m}(0)}\right) \cong 4 \times 2.4 \times 10^{-7} t^{3}-3 \times 4.3 \times 10^{-5} t^{2}+2 \times 0.0026 t-0.017$

\section{Parameterization Conditions and Assumptions}

i. The proportion of Males in consolidated recovery rates and death rates was assumed to be higher than their Female counter parts. This was based on premise that the number males in quarantine is higher than the Females. $\square_{\mathrm{M} 2} \geq \square_{F_{2}}$; $\square_{1} \geq$ $\square 2$.

ii. The enlisting rates of Infectious or individuals in latency state is assumed to be a nonnegative proper fraction. That is $0 \leq \square_{1} \leq 1 ; 0 \leq \square_{2} \leq 1$.

iii. The data was fitted on condition that individuals in community in Latency state are always greater or equal to those in infectious state. That is $l_{m} \geq i_{m}$ and $l_{f} \geq i_{f}$.

\section{RESULTS}

Equation (15) was fitted in observed data using nonlinear least square method to estimate the state variables $l_{m}$ and $i_{m}$ and parameters $h_{m}$ and $\square$. Equation (16) was fitted in observed data using nonlinear least square method to estimate the state variables $l_{f}$ and $i_{f}$ and parameters $h_{f}$ and $\square 2$ The daily estimates of parameters and state variables were summarized in Appendix 1 and Appendix 2. Note $\mathrm{E}$ in the Appendix 1 and Appendix 2 stand for powers of base 10. The total sum least square of the estimates for equation 15 and equation 16 were obtained as $1.9366 \times 10^{-9}$ and $9.83278 \times 10^{-5}$.

The graphical presentations were obtained below.

Figure 4: Fitting data of Males to Model

Figure 5: Fitting data of Females to Model

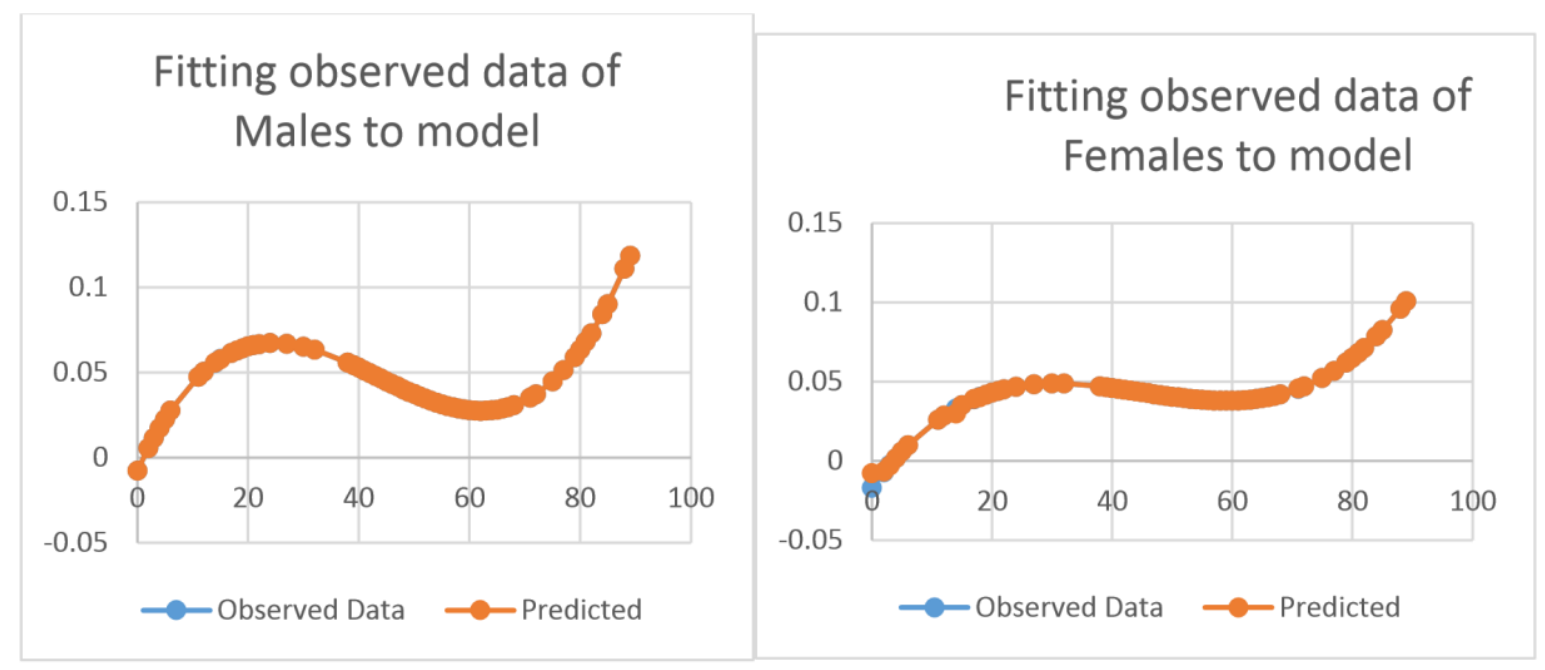

The results indicated that model fitted well to the data with varying estimates of parameters and 
state variables.

\section{Parameter Estimates}

The daily estimates of enlisting of Males and Females to quarantine centers was for 89 days as presented graphically below. Figure 6: Rate of enlisting of Males and Females to Quarantine centers Figure 7: Proportion of

enlisting individuals in Infectious and Latency state from Community

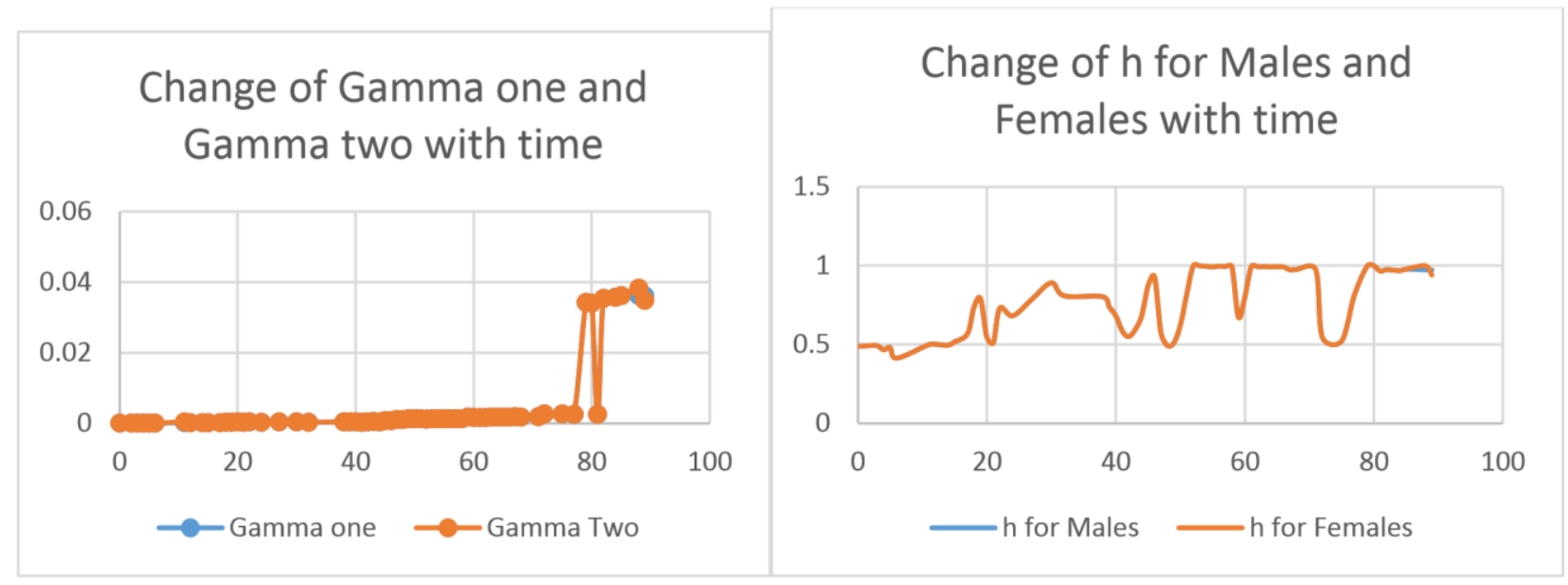

The result indicated similarity in rate of enlisting both genders to quarantine centers and in proportions of infectious and latent individuals drawn from community.

\section{State Estimates}

The daily estimates of infected Males and Females to quarantine centers was for 89 days as presented graphically below. Figure 8: Estimates of infected Males and Females in The Community Figure 9: Estimates of individuals (Males and Females) in Latency state in the community

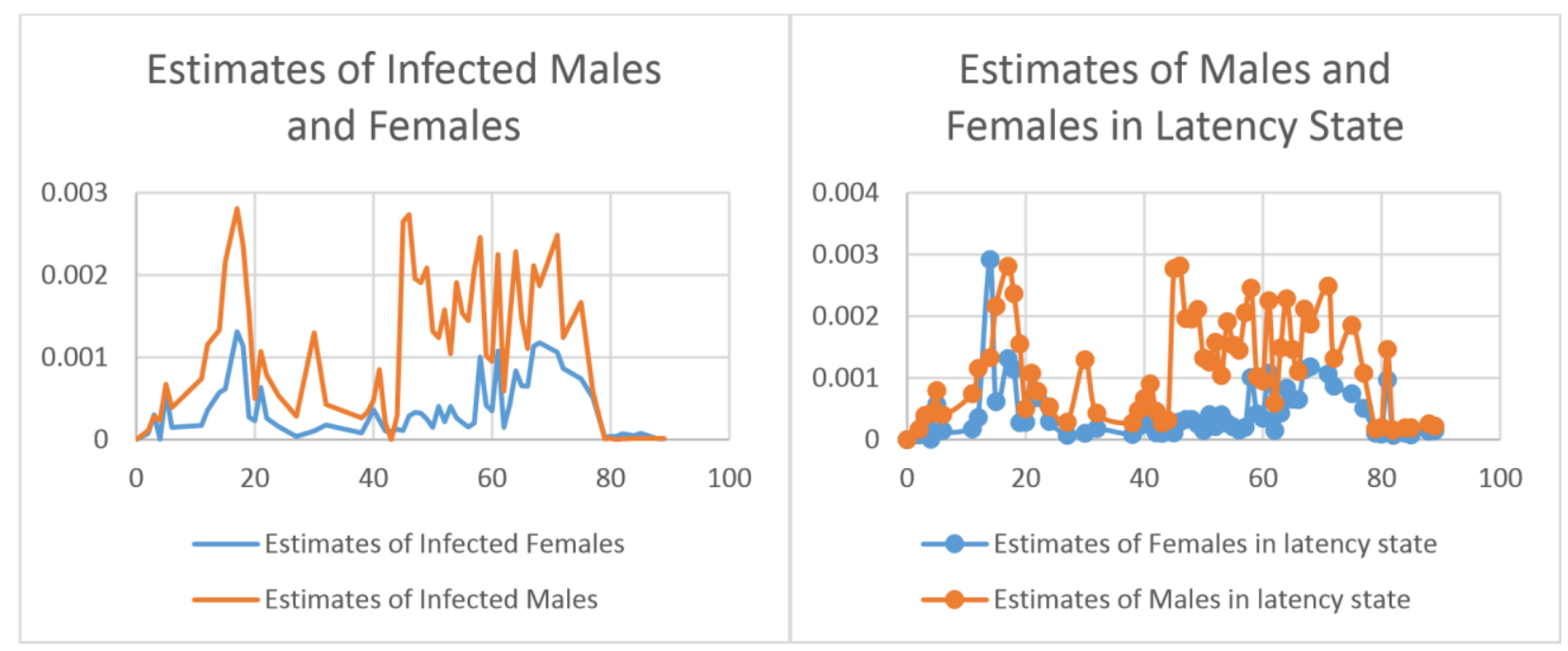

The results in Figure 8 indicates the number of infected males in the community has been higher than those of Females in 89 days. The maximum, Average and Minimum proportion of infected Males in the community were: $0.001313988,0.000367992$ and 1.66565E-07. The maximum,

Average and Minimum proportion of infected Females in the community were: 0.002808409 ,

0.00108494 and 0. The maximum, Average and Minimum proportion of latent Males in the community were: 0.002818108, 0.001167956 and 5.09596E-07. The maximum, Average and Minimum proportion of latent Females in the community were: $0.002921332,0.000437533$ and $1.66565 \mathrm{E}-07$. 


\section{4. DISCUSSION}

Our study used the estimates of the last five days (84th to $89^{\text {th }}$ day) to predict the dynamics of the individuals in quarantine. The tables 1 and 2 is the summary of the parameters and state variables from $84^{\text {th }}$ to $89^{\text {th }}$ day obtained from tables 4 and 5 . The best combination of values in table 1 and table 2 were used to simulate maximum and minimum possible number of males and Females in quarantine and their averages. Date 19/7/2020 (day 89) was used as initial condition of the model where $\mathrm{q}_{\mathrm{m}}$ and $\mathrm{q}_{\mathrm{f}}$ were $1.53304 \times 10^{-5}$ and $1.00772 \times 10^{-5}$ respectively. Matlab inbuilt ode solver was used to simulate the dynamics of equations 11 and 14 to obtain figure 10.

Figure 10: Predictions of dynamics of equations 11 and 14

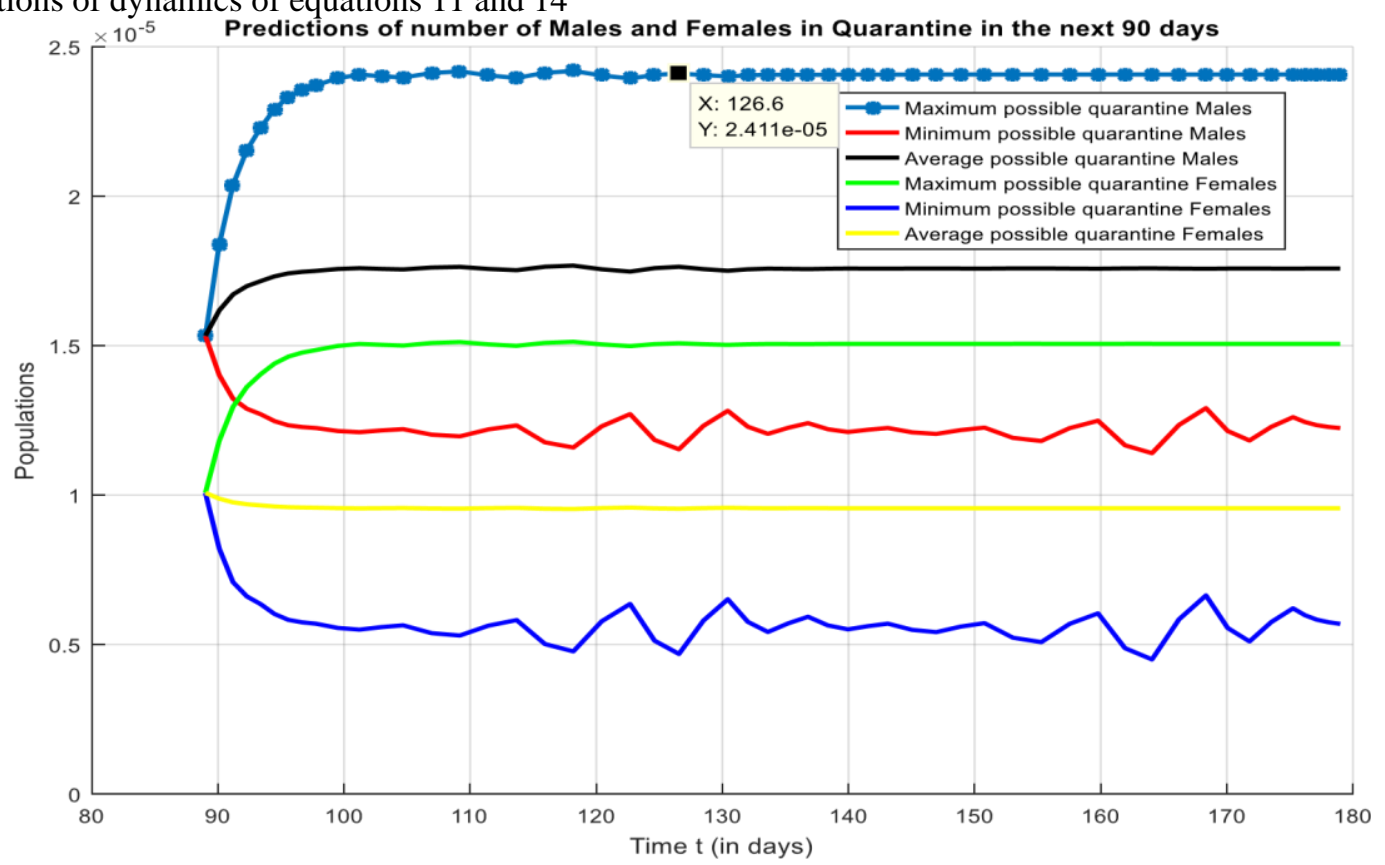

The results obtained in Figure 10 indicated maximum possible males and Females in Quarantine would be $2.406 \times 10^{-5}$ (567 Males) and $1.509 \times 10^{-5}(363$ Females $)$ would remain in quarantine after death and recovery by $120^{\text {th }}$ day and $107^{\text {th }}$ day respectively. The minimum possible males and Females in Quarantine would be $1.233 \times 10^{-5}$ (291 Males) and $4.774 \times$

$10^{-6}$ (116 Females) would remain in quarantine after death and recovery by $114^{\text {th }}$ day and $119^{\text {th }}$ respectively. The average Males and Females in Quarantine would be $1.747 \times 10^{-5}$ (412 Males) and $9.567 \times 10^{-6}(230$ Females $)$ would remain in quarantine after death and recovery by $97^{\text {th }}$ day and $132^{\text {th }}$ day.

\section{5. CONCLUSION}

COVID-19 Sex based model was formulated using first order ordinary differential equations and equations (1)-(14) obtained. Quarantine data from Ministry of health was used to estimate daily number of infected and latent individuals in the Kenya community and result summarized in Figures 8 and 9. The estimates indicates that Males are bearing more COVID-19 burden in the community. Figure 6 indicates the Government effort in enlisting individuals to Quarantine centers is not gender biased. Simulation indicates that the number of Males and Females who will remain constantly infected after disease induced death and recoveries ranges $(567-219)$ and $(363-116)$ for Males and females, respectively.

Future studies should consider Mathematical analysis of the model to determine well posed of the system, determine reproduction number and use it to determine stability of steady states. Although this study consider prediction of males and females in quarantine, future studies should strive to predict dynamics of the infectious and exposed in the community.

\section{REFERENCES}

[1] Abiad, A., Arao, R. M., \& Dagli, S. (2020). The Economic Impact of the COVID-19 Outbreak on Developing Asia. Mandaluyong: Asian Development Bank.

[2] Cascella, M., Rajnik, M., Cuomo, A., Dulebohn, S. C., \& Napoli, R. D. (2020). Features, Evaluation and Treatment Coronavirus (COVID-19). StatPearls Publishing.

[3] Centers for Disease Control and Prevention and Others. (2020). What you should Know about COVID-19 to Protect Yourself and Others. Washington, DC: Centers for Disease Control and Prevention.

[4] Erdos, P., \& Turan, P. (1940). On Interpolation. III. Interpolatory Theory of Polynomials. Annals of Mathematics, 510 - 553.

[5] Gill, P. E., \& Murray, W. (1978). Algorithms for the Solution of the Nonlinear LeastSquares Problem. SIAM Journal on Numerical Analysis, 977 $-992$.

[6] Golub, G., \& Pereyra, V. (2003). Separable Nonlinear Least Squares: The Variable Projection Method and Its Applications. Inverse Problems, R1.

[7] Johnson, M. L., \& Frasier, S. G. (1985). [16] Nonlinear Least-Squares Analysis. Methods in Enzymology, 301 - 342.

[8] Jr, J. E., Gay, D. M., \& Walsh, R. E. (1981). An Adaptive Nonlinear LeastSquares

[9] Algorithm. ACM Transactions on Mathematical Software (TOMS), 7(3), 348 -368 . 
[10] Philips, G. M. (2003). Interpolation and Approximation by Polynomials (Vol. 14). Berlin:

[11] Springer Science \& Business Media.

[12] Robin, A. (1972). Interactive Interpolation and Approximation by Bezier Polynomials. The British Computer Society, 15(1), 71 - 79.

[13] U.S. Department of Labor. (2020). Guidance on Preparing Workplaces for COVID-19. Washington, DC: Occupational Safety and Health Administration (OSHA)

[14] Velavan, T. P., \& Meyer, C. G. (2020). The COVID-19 Epidemic. Tropical medicine \& International Health, 25(3), 278.

[15] Worldometer. (2020, July 20). COVID-19 Coronavirus Pandemic. Retrieved from Worldometer: https://www.worldometers.info/coronavirus/

[16] Wu, C.-F. (1981). Asymptotic Theory of Nonlinear Least Squares Estimation. The Annals of Statistics, $501-513$.

[17] Zheng, Y.-Y., Ma, Y.-T., Zhang, J.-Y., \& Xie, X. (2020). COVID-19 and the Cardiovascular System. Nature Reviews Cardiology, 17(5), 259 - 260.

[18] Zippel, R. (1990). Interpolating Polynomials from Their Values. Journal of Symbolic Computation, 9(3), 375 - 403.

\section{AUTHORS}

First Author - Cyrus Gitonga Ngari, Department of Mathematics, Computing, and Information Technology; University of Embu

Second Author - Dominic Makaa Kitavi, Department of Mathematics, Computing, and Information Technology; University of Embu

Third Author - Paul Muriithi Ngari, Disease Surveillance and Response Unit; Embu Teaching and Referral Hospital Fourth Author - David Mugo Muchangi, Disease Surveillance and Response Unit; Embu Teaching and Referral Hospital

Corresponding Author Email: ngaripaul19@gmail.com

Table 1: Summary of Maximum, Minimum and average values of parameters and state variables of equation 11

\begin{tabular}{|c|c|c|c|c|c|c|}
\hline $\begin{array}{l}\text { Males } \\
\text { Parameter/ } \\
\text { State }\end{array}$ & $\boldsymbol{h}_{m}$ & ?1 & $i_{m}$ & $l_{m}$ & ? 1 & 国2 \\
\hline Maximum & 0.977659799 & 0.036172672 & $\begin{array}{l}1.83252 \mathrm{E}- \\
05\end{array}$ & 0.000255616 & 0.425503313 & 0.014006078 \\
\hline Minimum & 0.968475945 & 0.035417709 & $\begin{array}{l}1.08633 \mathrm{E}- \\
05\end{array}$ & 0.000155295 & 0.37407492 & 0.001469557 \\
\hline Average & 0.973637214 & 0.035931195 & $\begin{array}{l}1.44432 \mathrm{E}- \\
05\end{array}$ & 0.000202967 & 0.398302753 & 0.006358554 \\
\hline
\end{tabular}

Table 2: Summary of Maximum, Minimum and average values of parameters and state variables of equation 14

\begin{tabular}{|c|c|c|c|c|c|c|}
\hline $\begin{array}{l}\text { Females } \\
\text { Parameter/ } \\
\text { State }\end{array}$ & $\boldsymbol{h}_{f}$ & ?2 & $\boldsymbol{i}_{\boldsymbol{f}}$ & $l_{f}$ & ? 2 & 再2 \\
\hline Maximum & 0.99998268 & 0.038368687 & $\begin{array}{l}7.99106 \mathrm{E}- \\
05\end{array}$ & 0.000147418 & 0.425503313 & 0.014006078 \\
\hline Minimum & 0.941087668 & 0.034880943 & $\begin{array}{l}1.00764 \mathrm{E}- \\
05\end{array}$ & 7.38167E-05 & 0.37407492 & 0.001469557 \\
\hline Average & 0.972429689 & 0.036118515 & $\begin{array}{l}4.52846 \mathrm{E}- \\
05\end{array}$ & 0.000108868 & 0.398302753 & 0.006358554 \\
\hline
\end{tabular}

Table 3: Data from Ministry of Health

\begin{tabular}{|l|l|l|l|l|l|}
\hline DATE & $\begin{array}{l}\text { CUMMULATIVE } \\
\text { INFECTIONS }\end{array}$ & MALES & FEMALES & RECOVERIES & DEATHS \\
\hline $18 / 4 / 2020$ & 16 & 12 & 4 & 7 & 1 \\
\hline
\end{tabular}




\begin{tabular}{|c|c|c|c|c|c|}
\hline $20 / 4 / 2020$ & 27 & 6 & 5 & 2 & 0 \\
\hline $21 / 4 / 2020$ & 42 & 7 & 8 & 5 & 0 \\
\hline $22 / 4 / 2020$ & 49 & 5 & 2 & 9 & 0 \\
\hline $23 / 4 / 2020$ & 66 & 9 & 8 & 6 & 0 \\
\hline $24 / 4 / 2020$ & 82 & 7 & 9 & 5 & 0 \\
\hline $29 / 4 / 2020$ & 92 & 6 & 4 & 5 & 1 \\
\hline $30 / 4 / 2020$ & 104 & 9 & 3 & 15 & 2 \\
\hline $2 / 5 / 2020$ & 128 & 10 & 14 & 2 & 1 \\
\hline $3 / 5 / 2020$ & 158 & 23 & 7 & 15 & 2 \\
\hline $5 / 5 / 2020$ & 203 & 30 & 15 & 9 & 0 \\
\hline $6 / 5 / 2020$ & 250 & 31 & 16 & 8 & 2 \\
\hline $7 / 5 / 2020$ & 275 & 21 & 4 & 7 & 3 \\
\hline $8 / 5 / 2020$ & 283 & 9 & 5 & 5 & 0 \\
\hline $9 / 5 / 2020$ & 317 & 17 & 11 & 5 & 1 \\
\hline $10 / 5 / 2020$ & 340 & 13 & 10 & 32 & 2 \\
\hline $12 / 5 / 2020$ & 355 & 10 & 5 & 8 & 3 \\
\hline $15 / 5 / 2020$ & 378 & 18 & 5 & 0 & 3 \\
\hline $18 / 5 / 2020$ & 403 & 23 & 2 & 22 & 0 \\
\hline $20 / 5 / 2020$ & 469 & 43 & 23 & 8 & 0 \\
\hline $26 / 5 / 2020$ & 531 & 45 & 17 & 3 & 0 \\
\hline $27 / 5 / 2020$ & 654 & 78 & 45 & 3 & 3 \\
\hline $28 / 5 / 2020$ & 801 & 87 & 60 & 13 & 3 \\
\hline $30 / 5 / 2020$ & 944 & 110 & 33 & 26 & 1 \\
\hline $31 / 5 / 2020$ & 1018 & 55 & 19 & 14 & 1 \\
\hline $1 / 6 / 2020$ & 1077 & 35 & 24 & 8 & 5 \\
\hline $2 / 6 / 2020$ & 1149 & 50 & 22 & 17 & 2 \\
\hline $3 / 6 / 2020$ & 1272 & 90 & 33 & & 3 \\
\hline $4 / 6 / 2020$ & 1396 & 100 & 24 & 39 & 4 \\
\hline $5 / 6 / 2020$ & 1530 & 98 & 36 & 51 & 1 \\
\hline
\end{tabular}




\begin{tabular}{|c|c|c|c|c|c|}
\hline $6 / 6 / 2020$ & 1656 & 98 & 28 & 63 & 4 \\
\hline $7 / 6 / 2020$ & 1823 & 125 & 42 & 46 & 1 \\
\hline $9 / 6 / 2020$ & 1950 & 84 & 33 & 24 & 3 \\
\hline $10 / 6 / 2020$ & 2055 & 77 & 28 & 175 & 1 \\
\hline $11 / 6 / 2020$ & 2176 & 92 & 29 & 44 & 3 \\
\hline $12 / 6 / 2020$ & 2256 & 62 & 28 & 72 & 4 \\
\hline $13 / 6 / 2020$ & 2418 & 116 & 36 & 57 & 4 \\
\hline $15 / 6 / 2020$ & 2551 & 93 & 40 & 33 & 1 \\
\hline $16 / 6 / 2020$ & 2634 & 88 & 25 & 40 & 1 \\
\hline $17 / 6 / 2020$ & 2868 & 129 & 55 & 27 & 2 \\
\hline $18 / 6 / 2020$ & 3081 & 151 & 62 & 106 & 10 \\
\hline $19 / 6 / 2020$ & 3198 & 83 & 34 & 91 & 2 \\
\hline $20 / 6 / 2020$ & 3302 & 76 & 28 & 36 & 2 \\
\hline $21 / 6 / 2020$ & 3562 & 176 & 84 & 21 & 2 \\
\hline $22 / 6 / 2020$ & 3621 & 47 & 12 & 73 & 2 \\
\hline $23 / 6 / 2020$ & 3776 & 120 & 35 & 102 & 3 \\
\hline $24 / 6 / 2020$ & 4030 & 186 & 68 & 41 & 2 \\
\hline $25 / 6 / 2020$ & 4208 & 123 & 55 & 34 & 2 \\
\hline $26 / 6 / 2020$ & 4357 & 94 & 55 & 48 & 5 \\
\hline $27 / 6 / 2020$ & 4635 & 181 & 97 & 31 & 4 \\
\hline $28 / 6 / 2020$ & 4894 & 159 & 100 & 35 & 2 \\
\hline $1 / 7 / 2020$ & 5201 & 215 & 92 & 50 & 1 \\
\hline $2 / 7 / 2020$ & 5469 & 160 & 108 & 20 & 3 \\
\hline $5 / 7 / 2020$ & 5778 & 217 & 92 & 51 & 1 \\
\hline $7 / 7 / 2020$ & 5961 & 119 & 62 & 90 & 3 \\
\hline $9 / 7 / 2020$ & 6408 & 280 & 167 & 64 & 4 \\
\hline $10 / 7 / 2020$ & 6881 & 324 & 149 & 76 & 8 \\
\hline $11 / 7 / 2020$ & 7159 & 166 & 112 & 99 & 3 \\
\hline $12 / 7 / 2020$ & 7538 & 253 & 126 & 49 & 1 \\
\hline
\end{tabular}




\begin{tabular}{|l|l|l|l|l|l|}
\hline $14 / 7 / 2020$ & 8035 & 318 & 179 & 71 & 5 \\
\hline $15 / 7 / 2020$ & 8496 & 320 & 141 & 51 & 7 \\
\hline $18 / 7 / 2020$ & 9184 & 425 & 263 & 457 & 3 \\
\hline $19 / 7 / 2020$ & 9787 & 361 & 242 & 682 & 9 \\
\hline
\end{tabular}

Table 4: Fitting observed data of Males to model

\begin{tabular}{|c|c|c|c|c|c|c|c|c|c|}
\hline DATE & $\boldsymbol{h}_{\mathrm{m}}$ & $?_{1}$ & im & Im & 目 1 & ?2 & $\begin{array}{l}\text { Observed } \\
\text { Data }\end{array}$ & Predicted & $\begin{array}{l}\text { Least } \\
\text { square } \\
\text { error }\end{array}$ \\
\hline $\begin{array}{l}18 / 4 / 202 \\
0\end{array}$ & $\begin{array}{l}0.48874 \\
7\end{array}$ & $6.19 \mathrm{E}-05$ & $6.1 \mathrm{E}-07$ & $5.1 \mathrm{E}-07$ & 3.36E-05 & 0.00773472 & 0.0077 & $\begin{array}{l}- \\
0.00770019 \\
2\end{array}$ & $\begin{array}{l}3.70406 \mathrm{E} \\
-14\end{array}$ \\
\hline $\begin{array}{l}20 / 4 / 202 \\
0\end{array}$ & $\begin{array}{l}0.49353 \\
4 \\
\end{array}$ & $6.85 \mathrm{E}-05$ & 0.00011 & $\begin{array}{l}0.00017 \\
1 \\
\end{array}$ & $\begin{array}{l}0.01608 \\
1\end{array}$ & $\begin{array}{l}0.01599367 \\
8\end{array}$ & $\begin{array}{l}0.0055201 \\
6\end{array}$ & & $\begin{array}{l}8.90375 E \\
-14\end{array}$ \\
\hline $\begin{array}{l}21 / 4 / 202 \\
0\end{array}$ & $\begin{array}{l}0.49305 \\
5 \\
\end{array}$ & $6.53 \mathrm{E}-05$ & $\begin{array}{l}0.00028 \\
6 \\
\end{array}$ & $\begin{array}{l}0.00039 \\
3 \\
\end{array}$ & $\begin{array}{l}0.03147 \\
7 \\
\end{array}$ & $\begin{array}{l}0.03133759 \\
3\end{array}$ & $\begin{array}{l}0.0115590 \\
4\end{array}$ & & $\begin{array}{l}5.86039 \mathrm{E} \\
-13 \\
\end{array}$ \\
\hline $\begin{array}{l}22 / 4 / 202 \\
0\end{array}$ & & 0.0001 & & & & $\begin{array}{l}0.00611985 \\
3\end{array}$ & & & $20937 \mathrm{E}$ \\
\hline $\begin{array}{l}23 / 4 / 202 \\
0\end{array}$ & $\begin{array}{l}0.48088 \\
7\end{array}$ & 8.37E-05 & $\begin{array}{l}0.00067 \\
6\end{array}$ & & $\begin{array}{l}0.06923 \\
8 \\
\end{array}$ & $\begin{array}{l}0.06904519 \\
1\end{array}$ & & 02253895 & $8341 \mathrm{E}$ \\
\hline $\begin{array}{l}24 / 4 / 202 \\
0\end{array}$ & 0.41276 & $2.05 \mathrm{E}-05$ & $\begin{array}{l}0.00039 \\
2\end{array}$ & $\begin{array}{l}0.00040 \\
8\end{array}$ & $1.49 \mathrm{E}-07$ & 0 & $\begin{array}{l}0.0275003 \\
2\end{array}$ & 0.02750032 & $\begin{array}{l}58611 \mathrm{E} \\
0\end{array}$ \\
\hline $\begin{array}{l}29 / 4 / 202 \\
0\end{array}$ & $\begin{array}{l}0.49932 \\
7 \\
\end{array}$ & $\begin{array}{l}0.00017 \\
1\end{array}$ & $\begin{array}{l}0.00074 \\
3\end{array}$ & $\begin{array}{l}0.00074 \\
3\end{array}$ & $\begin{array}{l}0.39675 \\
4\end{array}$ & $\begin{array}{l}0.05587820 \\
2\end{array}$ & $\begin{array}{l}0.0473651 \\
2\end{array}$ & $\begin{array}{l}0.04736559 \\
2\end{array}$ & $\begin{array}{l}23033 \mathrm{E} \\
3\end{array}$ \\
\hline $\begin{array}{l}\text { 30/4/202 } \\
0\end{array}$ & $\begin{array}{l}0.50156 \\
3\end{array}$ & .00016 & $\begin{array}{l}0.00115 \\
5\end{array}$ & $\begin{array}{l}0.00115 \\
5\end{array}$ & $\begin{array}{l}0.37052 \\
8\end{array}$ & $\begin{array}{l}0.07882388 \\
3\end{array}$ & $\begin{array}{l}0.0504145 \\
6\end{array}$ & $\begin{array}{l}0.05042066 \\
7\end{array}$ & $12934 \mathrm{E}$ \\
\hline $2 / 5 / 2020$ & 49680 & $\begin{array}{l}0.00015 \\
9\end{array}$ & $\begin{array}{l}0.00133 \\
2\end{array}$ & $\begin{array}{l}0.00133 \\
2\end{array}$ & $\begin{array}{l}0.34696 \\
5\end{array}$ & $\begin{array}{l}0.097210 \\
2\end{array}$ & $\begin{array}{l}0.0556628 \\
8\end{array}$ & 57911 & $3606 \mathrm{E}$ \\
\hline $3 / 5 / 2020$ & $\begin{array}{l}0.51700 \\
8\end{array}$ & $\begin{array}{l}0.00022 \\
6\end{array}$ & $\begin{array}{l}0.00216 \\
1\end{array}$ & $\begin{array}{l}0.00216 \\
1\end{array}$ & 0.40613 & $\begin{array}{l}0.03611075 \\
1\end{array}$ & & 58 & $4221 E$ \\
\hline $5 / 5 / 2020$ & 0.5687 & $\begin{array}{l}0.00022 \\
7\end{array}$ & $\begin{array}{l}0.00280 \\
8\end{array}$ & $\begin{array}{l}0.00280 \\
8\end{array}$ & $\begin{array}{l}0.10235 \\
6\end{array}$ & $\begin{array}{l}0.33573183 \\
2\end{array}$ & $\begin{array}{l}0.0615457 \\
6\end{array}$ & $\begin{array}{l}0.06153100 \\
9\end{array}$ & $\begin{array}{l}2.17604 \mathrm{E} \\
-10\end{array}$ \\
\hline $6 / 5 / 2020$ & $\begin{array}{l}0.73686 \\
4 \\
\end{array}$ & $\begin{array}{l}0.00027 \\
8 \\
\end{array}$ & $\begin{array}{l}0.00236 \\
7 \\
\end{array}$ & $\begin{array}{l}0.00236 \\
7 \\
\end{array}$ & $\begin{array}{l}0.39624 \\
4 \\
\end{array}$ & $\begin{array}{l}0.04065878 \\
1\end{array}$ & $\begin{array}{l}0.0630126 \\
4\end{array}$ & $\begin{array}{l}0.06301555 \\
5\end{array}$ & $\begin{array}{l}8.49863 \mathrm{E} \\
-12 \\
\end{array}$ \\
\hline $7 / 5 / 2020$ & $\begin{array}{l}0.79160 \\
6\end{array}$ & $\begin{array}{l}0.00028 \\
7\end{array}$ & $\begin{array}{l}0.00155 \\
3\end{array}$ & $\begin{array}{l}0.00155 \\
3\end{array}$ & $\begin{array}{l}0.34002 \\
6\end{array}$ & $\begin{array}{l}0.09600405 \\
4\end{array}$ & $\begin{array}{l}0.0642476 \\
8\end{array}$ & $\begin{array}{l}0.06424360 \\
4\end{array}$ & $\begin{array}{l}1.66132 \mathrm{E} \\
-11\end{array}$ \\
\hline $8 / 5 / 2020$ & .54608 & $\begin{array}{l}0.00 \\
1\end{array}$ & $\begin{array}{l}0.00050 \\
2\end{array}$ & $\begin{array}{l}0.00050 \\
2\end{array}$ & $\begin{array}{l}0.18650 \\
8\end{array}$ & $\begin{array}{l}0.24823408 \\
9\end{array}$ & 0.06526 & .06525756 & $23848 \mathrm{E}$ \\
\hline $9 / 5 / 2020$ & $\begin{array}{l}0.50988 \\
6\end{array}$ & $\begin{array}{l}0.00033 \\
6\end{array}$ & $\begin{array}{l}0.00107 \\
6\end{array}$ & $\begin{array}{l}0.00107 \\
6\end{array}$ & $\begin{array}{l}0.40368 \\
8\end{array}$ & $\begin{array}{l}0.03043600 \\
7\end{array}$ & $\begin{array}{l}0.0660587 \\
2\end{array}$ & $\begin{array}{l}0.06604557 \\
1\end{array}$ & $\begin{array}{l}1.729 \mathrm{E}- \\
10\end{array}$ \\
\hline $\begin{array}{l}10 / 5 / 202 \\
0\end{array}$ & $\begin{array}{l}0.73080 \\
5\end{array}$ & $\begin{array}{l}0.00035 \\
1\end{array}$ & $\begin{array}{l}0.00078 \\
6 \\
\end{array}$ & $\begin{array}{l}0.00078 \\
6\end{array}$ & $\begin{array}{l}0.36381 \\
4\end{array}$ & $\begin{array}{l}0.06952249 \\
5\end{array}$ & $\begin{array}{l}0.0666529 \\
6\end{array}$ & $\begin{array}{l}0.06665525 \\
2\end{array}$ & $\begin{array}{l}5.25383 \mathrm{E} \\
-12\end{array}$ \\
\hline $\begin{array}{l}12 / 5 / 202 \\
0\end{array}$ & $\begin{array}{l}0.68156 \\
4\end{array}$ & $\begin{array}{l}0.00034 \\
3\end{array}$ & $\begin{array}{l}0.00053 \\
7\end{array}$ & $\begin{array}{l}0.00053 \\
7\end{array}$ & $\begin{array}{l}0.31928 \\
5\end{array}$ & $\begin{array}{l}0.04736740 \\
1\end{array}$ & $\begin{array}{l}0.0672644 \\
8\end{array}$ & $\begin{array}{l}0.06726560 \\
7\end{array}$ & $\begin{array}{l}1.26931 \mathrm{E} \\
-12\end{array}$ \\
\hline $\begin{array}{l}15 / 5 / 202 \\
0\end{array}$ & $\begin{array}{l}0.78778 \\
9\end{array}$ & $\begin{array}{l}0.00035 \\
7\end{array}$ & $\begin{array}{l}0.00028 \\
3\end{array}$ & $\begin{array}{l}0.00028 \\
3\end{array}$ & $\begin{array}{l}0.06056 \\
9\end{array}$ & $\begin{array}{l}0.00463003 \\
4\end{array}$ & $\begin{array}{l}0.0668761 \\
6\end{array}$ & $\begin{array}{l}0.06687502 \\
9\end{array}$ & $\begin{array}{l}1.27963 \mathrm{E} \\
-12\end{array}$ \\
\hline $\begin{array}{l}18 / 5 / 202 \\
0\end{array}$ & $\begin{array}{l}0.89190 \\
6\end{array}$ & $\begin{array}{l}0.00037 \\
9\end{array}$ & 0.0013 & 0.0013 & 0.02914 & $\begin{array}{l}0.41077191 \\
2\end{array}$ & 0.06514 & $\begin{array}{l}0.06513999 \\
8\end{array}$ & $\begin{array}{l}2.62398 \mathrm{E} \\
-18\end{array}$ \\
\hline $\begin{array}{l}20 / 5 / 202 \\
0\end{array}$ & $\begin{array}{l}0.80835 \\
7\end{array}$ & $\begin{array}{l}0.00030 \\
5\end{array}$ & $\begin{array}{l}0.00042 \\
7 \\
\end{array}$ & $\begin{array}{l}0.00043 \\
3 \\
\end{array}$ & $\begin{array}{l}0.00872 \\
3\end{array}$ & 0 & $\begin{array}{l}0.0633553 \\
6\end{array}$ & $\begin{array}{l}0.06332395 \\
4\end{array}$ & $\begin{array}{l}9.86354 \mathrm{E} \\
-10\end{array}$ \\
\hline
\end{tabular}

This publication is licensed under Creative Commons Attribution CC BY. 


\begin{tabular}{|c|c|c|c|c|c|c|c|c|c|}
\hline $\begin{array}{l}26 / 5 / 202 \\
0\end{array}$ & $\begin{array}{l}0.80327 \\
3\end{array}$ & $\begin{array}{l}0.00041 \\
4\end{array}$ & $\begin{array}{l}0.00026 \\
5\end{array}$ & $\begin{array}{l}0.00027 \\
3\end{array}$ & $\begin{array}{l}0.00294 \\
6\end{array}$ & $\begin{array}{l}0.00014393 \\
7\end{array}$ & $\begin{array}{l}0.0557934 \\
4\end{array}$ & $\begin{array}{l}0.0557926 \\
4\end{array}$ & $\begin{array}{l}5.57135 \mathrm{E} \\
-13\end{array}$ \\
\hline $\begin{array}{l}27 / 5 / 202 \\
0\end{array}$ & $\begin{array}{l}0.73622 \\
1\end{array}$ & $\begin{array}{l}0.00044 \\
4\end{array}$ & 0.00034 & $\begin{array}{l}0.00047 \\
5\end{array}$ & $\begin{array}{l}0.00229 \\
4\end{array}$ & $\begin{array}{l}0.00229352 \\
3\end{array}$ & $\begin{array}{l}0.0543068 \\
8\end{array}$ & $\begin{array}{l}0.05430683 \\
7\end{array}$ & $\begin{array}{l}1.813 \mathrm{E}- \\
15\end{array}$ \\
\hline $\begin{array}{l}28 / 5 / 202 \\
0\end{array}$ & 0.68123 & .00038 & $\begin{array}{l}0.00047 \\
7\end{array}$ & $\begin{array}{l}0.00067 \\
2\end{array}$ & $\begin{array}{l}0.00811 \\
5 \\
\end{array}$ & $\begin{array}{l}0.00187265 \\
5\end{array}$ & 0.05278 & $\begin{array}{l}0.05277992 \\
9\end{array}$ & $\begin{array}{l}5.10804 \mathrm{E} \\
-15\end{array}$ \\
\hline $\begin{array}{l}30 / 5 / 202 \\
0\end{array}$ & $\begin{array}{l}0.58529 \\
6\end{array}$ & $\begin{array}{l}0.00034 \\
8\end{array}$ & $\begin{array}{l}0.00085 \\
2\end{array}$ & $\begin{array}{l}0.00090 \\
5\end{array}$ & $\begin{array}{l}0.01398 \\
3\end{array}$ & $\begin{array}{l}0.00052997 \\
5\end{array}$ & & $\begin{array}{l}0.05121994 \\
4\end{array}$ & $\begin{array}{l}3.90408 \mathrm{E} \\
-12\end{array}$ \\
\hline $\begin{array}{l}31 / 5 / 202 \\
0\end{array}$ & $\begin{array}{l}0.55005 \\
8\end{array}$ & $\begin{array}{l}0.00038 \\
3\end{array}$ & $\begin{array}{l}0.00020 \\
5\end{array}$ & $\begin{array}{l}0.00046 \\
5\end{array}$ & $\begin{array}{l}0.00694 \\
8\end{array}$ & $\begin{array}{l}0.00049152 \\
2\end{array}$ & $\begin{array}{l}0.0496417 \\
6\end{array}$ & $\begin{array}{l}0.04963789 \\
6\end{array}$ & $\begin{array}{l}1.49293 \mathrm{E} \\
-11\end{array}$ \\
\hline $1 / 6 / 2020$ & $\begin{array}{l}0.59445 \\
8\end{array}$ & $\begin{array}{l}0.00049 \\
4\end{array}$ & 0 & $\begin{array}{l}0.00027 \\
4\end{array}$ & $\begin{array}{l}0.00369 \\
7\end{array}$ & $\begin{array}{l}0.00231455 \\
9\end{array}$ & $\begin{array}{l}0.0480486 \\
4\end{array}$ & $\begin{array}{l}0.04804934 \\
3\end{array}$ & $\begin{array}{l}4.93717 \mathrm{E} \\
-13\end{array}$ \\
\hline $2 / 6 / 2020$ & $\begin{array}{l}0.68106 \\
1\end{array}$ & $\begin{array}{l}0.00037 \\
5\end{array}$ & $\begin{array}{l}0.00030 \\
2\end{array}$ & $\begin{array}{l}0.00031 \\
3\end{array}$ & $\begin{array}{l}0.00739 \\
8\end{array}$ & $\begin{array}{l}0.00087032 \\
2\end{array}$ & $\begin{array}{l}0.0464516 \\
8\end{array}$ & $\begin{array}{l}0.04645168 \\
3\end{array}$ & $\begin{array}{l}11007 \mathrm{E} \\
7\end{array}$ \\
\hline $3 / 6 / 2020$ & $\begin{array}{l}0.87189 \\
1\end{array}$ & $\begin{array}{l}0.00069 \\
3\end{array}$ & $\begin{array}{l}0.00265 \\
2\end{array}$ & $\begin{array}{l}0.00277 \\
1\end{array}$ & 0.44294 & 0.01226411 & 0.04486 & $\begin{array}{l}0.04486043 \\
7\end{array}$ & $\begin{array}{l}1.90832 \mathrm{E} \\
-13\end{array}$ \\
\hline $4 / 6 / 2020$ & $\begin{array}{l}0.92986 \\
8\end{array}$ & $\begin{array}{l}0.00075 \\
5\end{array}$ & $\begin{array}{l}0.00273 \\
5\end{array}$ & $\begin{array}{l}0.00281 \\
8\end{array}$ & $\begin{array}{l}0.43770 \\
6\end{array}$ & $\begin{array}{l}0.0190683 \\
7\end{array}$ & $\begin{array}{l}0.0432827 \\
2\end{array}$ & $\begin{array}{l}0.04328310 \\
4\end{array}$ & $\begin{array}{l}47174 \mathrm{E} \\
3\end{array}$ \\
\hline $5 / 6 / 2020$ & $\begin{array}{l}0.57133 \\
2\end{array}$ & $\begin{array}{l}0.00106 \\
1\end{array}$ & 0.00196 & & & 0.0051929 & & $\begin{array}{l}0.04172949 \\
1\end{array}$ & $1642 \mathrm{E}$ \\
\hline $6 / 6 / 2020$ & $\begin{array}{l}0.49238 \\
6\end{array}$ & $\begin{array}{l}0.00107 \\
6\end{array}$ & 0.00191 & $\begin{array}{l}0.00195 \\
9\end{array}$ & $\begin{array}{l}0.43560 \\
6\end{array}$ & $\begin{array}{l}0.02419556 \\
2\end{array}$ & $\begin{array}{l}0.0402078 \\
4\end{array}$ & $\begin{array}{l}0.04020815 \\
7\end{array}$ & $0577 \mathrm{E}$ \\
\hline $7 / 6 / 2020$ & $\begin{array}{l}0.50993 \\
6\end{array}$ & $\begin{array}{l}0.00126 \\
4\end{array}$ & $\begin{array}{l}0.00208 \\
9\end{array}$ & $\begin{array}{l}0.00210 \\
9\end{array}$ & $\begin{array}{l}0.45637 \\
4\end{array}$ & $\begin{array}{l}0.00482533 \\
1\end{array}$ & $\begin{array}{l}0.0387284 \\
8\end{array}$ & $\begin{array}{l}0.038729 \\
7\end{array}$ & $\begin{array}{l}3.68464 \mathrm{E} \\
-13\end{array}$ \\
\hline $9 / 6 / 2020$ & $\begin{array}{l}0.62185 \\
2\end{array}$ & 0.00135 & $\begin{array}{l}0.00132 \\
1\end{array}$ & $\begin{array}{l}0.00132 \\
2\end{array}$ & $\begin{array}{l}0.44542 \\
8\end{array}$ & $\begin{array}{l}0.01728583 \\
8\end{array}$ & 0.0373 & $\begin{array}{l}0.03730044 \\
6\end{array}$ & $\begin{array}{l}1.9857 \mathrm{E}- \\
13\end{array}$ \\
\hline $\begin{array}{l}10 / 6 / 202 \\
0\end{array}$ & $\begin{array}{l}0.82097 \\
4\end{array}$ & $\begin{array}{l}0.00130 \\
4\end{array}$ & $\begin{array}{l}0.00124 \\
4\end{array}$ & $\begin{array}{l}0.00125 \\
6\end{array}$ & $\begin{array}{l}0.45938 \\
2\end{array}$ & $\begin{array}{l}0.00473712 \\
7\end{array}$ & $\begin{array}{l}0.0359315 \\
2\end{array}$ & $\begin{array}{l}0.03593162 \\
8\end{array}$ & $15982 \mathrm{E}$ \\
\hline $\begin{array}{l}11 / 6 / 202 \\
0\end{array}$ & $\begin{array}{l}0.99992 \\
7\end{array}$ & $\begin{array}{l}0.00123 \\
9\end{array}$ & $\begin{array}{l}0.00157 \\
6\end{array}$ & $\begin{array}{l}0.00157 \\
6\end{array}$ & $\begin{array}{l}0.43 \\
4\end{array}$ & $\begin{array}{l}0.0257032 \\
8\end{array}$ & $\begin{array}{l}0.034 \\
6\end{array}$ & $\begin{array}{l}0.034 \\
1\end{array}$ & $6071 \mathrm{E}$ \\
\hline $\begin{array}{l}12 / 6 / 202 \\
0\end{array}$ & $\begin{array}{l}0.99928 \\
8\end{array}$ & $\begin{array}{l}0.00126 \\
3\end{array}$ & $\begin{array}{l}0.00104 \\
3\end{array}$ & $\begin{array}{l}0.00104 \\
3\end{array}$ & $\begin{array}{l}0.42377 \\
9\end{array}$ & 0.042 & $\begin{array}{l}0.0334110 \\
4\end{array}$ & 0.03 & $3762 \mathrm{E}$ \\
\hline $\begin{array}{l}13 / 6 / 202 \\
0\end{array}$ & $\begin{array}{l}0.99607 \\
9\end{array}$ & $\begin{array}{l}0.00128 \\
9\end{array}$ & $\begin{array}{l}0.00191 \\
1\end{array}$ & $\begin{array}{l}0.00191 \\
1\end{array}$ & $\begin{array}{l}0.44140 \\
8\end{array}$ & $\begin{array}{l}0.02633943 \\
5\end{array}$ & $\begin{array}{l}0.0322772 \\
8\end{array}$ & $\begin{array}{l}0.03227719 \\
6\end{array}$ & $\begin{array}{l}7.03961 \mathrm{E} \\
-15\end{array}$ \\
\hline $\begin{array}{l}15 / 6 / 202 \\
0\end{array}$ & $\begin{array}{l}0.99286 \\
8\end{array}$ & $\begin{array}{l}0.00128 \\
8\end{array}$ & $\begin{array}{l}0.00153 \\
3\end{array}$ & $\begin{array}{l}0.00153 \\
3\end{array}$ & $\begin{array}{l}0.46083 \\
6\end{array}$ & $\begin{array}{l}0.00795653 \\
6\end{array}$ & 0.03124 & $\begin{array}{l}0.03124020 \\
8\end{array}$ & $\begin{array}{l}4.32944 \mathrm{E} \\
-14\end{array}$ \\
\hline $\begin{array}{l}16 / 6 / 202 \\
0\end{array}$ & $\begin{array}{l}0.99633 \\
3\end{array}$ & $\begin{array}{l}0.00128 \\
8\end{array}$ & $\begin{array}{l}0.00145 \\
1\end{array}$ & $\begin{array}{l}0.00145 \\
1\end{array}$ & $\begin{array}{l}0.46372 \\
2\end{array}$ & 0.00588737 & $\begin{array}{l}0.0303083 \\
2\end{array}$ & $\begin{array}{l}0.03030827 \\
3\end{array}$ & $\begin{array}{l}2.21037 \mathrm{E} \\
-15\end{array}$ \\
\hline $\begin{array}{l}17 / 6 / 202 \\
0\end{array}$ & $\begin{array}{l}0.99488 \\
8\end{array}$ & 0.00133 & $\begin{array}{l}0.00205 \\
9\end{array}$ & $\begin{array}{l}0.00205 \\
9\end{array}$ & $\begin{array}{l}0.45523 \\
9\end{array}$ & $\begin{array}{l}0.01525332 \\
6\end{array}$ & $\begin{array}{l}0.02 \\
6\end{array}$ & $\begin{array}{l}0.029 \\
1\end{array}$ & $2677 \mathrm{E}$ \\
\hline $\begin{array}{l}18 / 6 / 202 \\
0\end{array}$ & $\begin{array}{l}0.99406 \\
5\end{array}$ & $\begin{array}{l}0.00130 \\
4\end{array}$ & $\begin{array}{l}0.00245 \\
9\end{array}$ & $\begin{array}{l}0.00245 \\
9\end{array}$ & $\begin{array}{l}0.44596 \\
4\end{array}$ & 0.02523591 & $\begin{array}{l}0.0287982 \\
4\end{array}$ & $\begin{array}{l}0.02879831 \\
5\end{array}$ & $\begin{array}{l}5.68324 \mathrm{E} \\
-15\end{array}$ \\
\hline $\begin{array}{l}19 / 6 / 202 \\
0\end{array}$ & $\begin{array}{l}0.67439 \\
6\end{array}$ & $\begin{array}{l}0.00172 \\
2\end{array}$ & $\begin{array}{l}0.00102 \\
3\end{array}$ & $\begin{array}{l}0.00102 \\
3\end{array}$ & $\begin{array}{l}0.46316 \\
9\end{array}$ & $\begin{array}{l}0.00858296 \\
8\end{array}$ & $\begin{array}{l}0.0282380 \\
8\end{array}$ & $\begin{array}{l}0.02823795 \\
7\end{array}$ & $\begin{array}{l}1.51485 E \\
-14\end{array}$ \\
\hline $\begin{array}{l}20 / 6 / 202 \\
0\end{array}$ & $\begin{array}{l}0.80035 \\
7\end{array}$ & $\begin{array}{l}0.00169 \\
6\end{array}$ & $\begin{array}{l}0.00095 \\
1\end{array}$ & $\begin{array}{l}0.00095 \\
1\end{array}$ & $\begin{array}{l}0.46165 \\
9\end{array}$ & $\begin{array}{l}0.01050614 \\
6\end{array}$ & 0.02782 & $\begin{array}{l}0.02781985 \\
5\end{array}$ & $\begin{array}{l}2.0926 \mathrm{E}- \\
14\end{array}$ \\
\hline $\begin{array}{l}21 / 6 / 202 \\
0\end{array}$ & $\begin{array}{l}0.99462 \\
8\end{array}$ & $\begin{array}{l}0.00165 \\
9\end{array}$ & $\begin{array}{l}0.00225 \\
3\end{array}$ & $\begin{array}{l}0.00225 \\
3\end{array}$ & $\begin{array}{l}0.46472 \\
1\end{array}$ & 0.00769502 & $\begin{array}{l}0.0275531 \\
2\end{array}$ & $\begin{array}{l}0.02755291 \\
9\end{array}$ & $\begin{array}{l}4.03814 \mathrm{E} \\
-14\end{array}$ \\
\hline $\begin{array}{l}22 / 6 / 202 \\
0\end{array}$ & $\begin{array}{l}0.99361 \\
8\end{array}$ & $\begin{array}{l}0.00168 \\
4\end{array}$ & $\begin{array}{l}0.00059 \\
2\end{array}$ & $\begin{array}{l}0.00059 \\
2\end{array}$ & $\begin{array}{l}0.45573 \\
8\end{array}$ & $\begin{array}{l}0.01682726 \\
1\end{array}$ & $\begin{array}{l}0.0274465 \\
6\end{array}$ & $\begin{array}{l}0.02744636 \\
5\end{array}$ & $\begin{array}{l}3.80463 E \\
-14\end{array}$ \\
\hline $\begin{array}{l}23 / 6 / 202 \\
0\end{array}$ & $\begin{array}{l}0.99460 \\
1\end{array}$ & $\begin{array}{l}0.00171 \\
3\end{array}$ & $\begin{array}{l}0.00148 \\
7\end{array}$ & $\begin{array}{l}0.00148 \\
7\end{array}$ & $\begin{array}{l}0.46267 \\
7\end{array}$ & $\begin{array}{l}0.00979312 \\
3\end{array}$ & $\begin{array}{l}0.0275094 \\
4\end{array}$ & $\begin{array}{l}0.02750928 \\
9\end{array}$ & $\begin{array}{l}2.27635 E \\
-14\end{array}$ \\
\hline $\begin{array}{l}24 / 6 / 202 \\
0\end{array}$ & $\begin{array}{l}0.99276 \\
2\end{array}$ & $\begin{array}{l}0.00172 \\
9\end{array}$ & $\begin{array}{l}0.00228 \\
4\end{array}$ & $\begin{array}{l}0.00228 \\
4\end{array}$ & $\begin{array}{l}0.46737 \\
2\end{array}$ & $\begin{array}{l}0.00485709 \\
8\end{array}$ & $\begin{array}{l}0.0277508 \\
8\end{array}$ & 0.02775073 & $\begin{array}{l}2.23509 E \\
-14\end{array}$ \\
\hline
\end{tabular}

This publication is licensed under Creative Commons Attribution CC BY. 


\begin{tabular}{|c|c|c|c|c|c|c|c|c|c|}
\hline $\begin{array}{l}25 / 6 / 202 \\
0\end{array}$ & $\begin{array}{l}0.99311 \\
1 \\
\end{array}$ & $\begin{array}{l}0.00178 \\
2 \\
\end{array}$ & $\begin{array}{l}0.00146 \\
6 \\
\end{array}$ & $\begin{array}{l}0.00146 \\
6 \\
\end{array}$ & $\begin{array}{l}0.46489 \\
4 \\
\end{array}$ & $\begin{array}{l}0.00689616 \\
3\end{array}$ & 0.02818 & 0.02817985 & $\begin{array}{l}2.24993 E \\
-14 \\
\end{array}$ \\
\hline $\begin{array}{l}26 / 6 / 202 \\
0\end{array}$ & $\begin{array}{l}0.99219 \\
4\end{array}$ & $\begin{array}{l}0.00180 \\
4\end{array}$ & $\begin{array}{l}0.00110 \\
6\end{array}$ & $\begin{array}{l}0.00110 \\
6 \\
\end{array}$ & $\begin{array}{l}0.45126 \\
9 \\
\end{array}$ & $\begin{array}{l}0.01992755 \\
6\end{array}$ & $\begin{array}{l}0.0288059 \\
2\end{array}$ & $\begin{array}{l}0.02880577 \\
5\end{array}$ & $\begin{array}{l}2.10219 E \\
-14\end{array}$ \\
\hline $\begin{array}{l}27 / 6 / 202 \\
0\end{array}$ & $\begin{array}{l}0.97388 \\
9\end{array}$ & & $\begin{array}{l}0.00211 \\
7\end{array}$ & $\begin{array}{l}0.00211 \\
7\end{array}$ & $\begin{array}{l}0.45650 \\
1\end{array}$ & $\begin{array}{l}0.01386643 \\
3\end{array}$ & & $\begin{array}{l}0.02963761 \\
6\end{array}$ & $\begin{array}{l}2.07275 E \\
-14\end{array}$ \\
\hline $\begin{array}{l}28 / 6 / 202 \\
0\end{array}$ & $\begin{array}{l}0.97562 \\
4\end{array}$ & & $\begin{array}{l}0.00187 \\
2\end{array}$ & $\begin{array}{l}0.00187 \\
2\end{array}$ & 0.4642 & $\begin{array}{l}0.00515614 \\
2\end{array}$ & $\begin{array}{l}0.0306846 \\
4\end{array}$ & $\begin{array}{l}0.03068464 \\
6\end{array}$ & $\begin{array}{l}3.2043 \mathrm{E}- \\
17\end{array}$ \\
\hline $1 / 7 / 2020$ & $\begin{array}{l}0.97362 \\
9\end{array}$ & $\begin{array}{l}0.00183 \\
4\end{array}$ & 0.00249 & 0.00249 & $\begin{array}{l}0.46298 \\
7 \\
\end{array}$ & $\begin{array}{l}0.00178128 \\
8\end{array}$ & $\begin{array}{l}0.0352067 \\
2\end{array}$ & $\begin{array}{l}0.03520673 \\
3\end{array}$ & $\begin{array}{l}1.7496 \mathrm{E}- \\
16\end{array}$ \\
\hline $2 / 7 / 2020$ & $\begin{array}{l}0.54772 \\
4\end{array}$ & $\begin{array}{l}0.00264 \\
7 \\
\end{array}$ & $\begin{array}{l}0.00124 \\
2\end{array}$ & $\begin{array}{l}0.00131 \\
7 \\
\end{array}$ & $\begin{array}{l}0.44798 \\
1 \\
\end{array}$ & $\begin{array}{l}0.01483711 \\
5\end{array}$ & $\begin{array}{l}0.0372049 \\
6\end{array}$ & $\begin{array}{l}0.03720490 \\
5\end{array}$ & $\begin{array}{l}2.98385 E \\
-15 \\
\end{array}$ \\
\hline $5 / 7 / 2020$ & $\begin{array}{l}0.52096 \\
7 \\
\end{array}$ & $\begin{array}{l}0.00261 \\
2\end{array}$ & $\begin{array}{l}0.00166 \\
9\end{array}$ & $\begin{array}{l}0.00185 \\
2 \\
\end{array}$ & $\begin{array}{l}0.45348 \\
1\end{array}$ & $\begin{array}{l}0.00176074 \\
7\end{array}$ & 0.0448 & 0.04479983 & $\begin{array}{l}2.89878 \mathrm{E} \\
-14\end{array}$ \\
\hline $7 / 7 / 2020$ & $\begin{array}{l}0.81501 \\
3\end{array}$ & $\begin{array}{l}0.00256 \\
4\end{array}$ & $\begin{array}{l}0.00058 \\
7 \\
\end{array}$ & $\begin{array}{l}0.00107 \\
6 \\
\end{array}$ & $\begin{array}{l}0.44026 \\
3 \\
\end{array}$ & $\begin{array}{l}0.00841273 \\
8\end{array}$ & $\begin{array}{l}0.0512881 \\
6\end{array}$ & $\begin{array}{l}0.05128824 \\
3\end{array}$ & $\begin{array}{l}6.94855 E \\
-15\end{array}$ \\
\hline $9 / 7 / 2020$ & $\begin{array}{l}0.99942 \\
9\end{array}$ & $\begin{array}{l}0.03425 \\
5\end{array}$ & $1.2 \mathrm{E}-05$ & $\begin{array}{l}0.00017 \\
4\end{array}$ & $\begin{array}{l}0.43506 \\
2\end{array}$ & $\begin{array}{l}0.00589410 \\
6\end{array}$ & 0590012 & 0130 & $5023 \mathrm{E}$ \\
\hline $\begin{array}{l}10 / 7 / 202 \\
0\end{array}$ & 1 & $\begin{array}{l}0.03406 \\
5\end{array}$ & $1.38 \mathrm{E}-05$ & $\begin{array}{l}0.00020 \\
2 \\
\end{array}$ & $\begin{array}{l}0.42420 \\
1 \\
\end{array}$ & 0.01226859 & 6334 & $\begin{array}{l}0.06333985 \\
2\end{array}$ & $\begin{array}{l}2.19301 \mathrm{E} \\
-14\end{array}$ \\
\hline $\begin{array}{l}11 / 7 / 202 \\
0\end{array}$ & $\begin{array}{l}0.96560 \\
6 \\
\end{array}$ & $\begin{array}{l}0.00249 \\
1 \\
\end{array}$ & 0 & $\begin{array}{l}0.00146 \\
6 \\
\end{array}$ & $\begin{array}{l}0.42636 \\
7 \\
\end{array}$ & $\begin{array}{l}0.00562370 \\
4 \\
\end{array}$ & $\begin{array}{l}0.0680123 \\
2 \\
\end{array}$ & $\begin{array}{l}0.06801236 \\
3 \\
\end{array}$ & $\begin{array}{l}1.89032 \mathrm{E} \\
-15 \\
\end{array}$ \\
\hline $\begin{array}{l}12 / 7 / 202 \\
0\end{array}$ & $\begin{array}{l}0.97494 \\
2 \\
\end{array}$ & $\begin{array}{l}0.03541 \\
8 \\
\end{array}$ & 1.09E-05 & $\begin{array}{l}0.00015 \\
5 \\
\end{array}$ & $\begin{array}{l}0.42550 \\
3 \\
\end{array}$ & $\begin{array}{l}0.00146955 \\
7\end{array}$ & $\begin{array}{l}0.0730273 \\
6\end{array}$ & $\begin{array}{l}0.07302740 \\
9\end{array}$ & $\begin{array}{l}2.3841 \mathrm{E}- \\
15\end{array}$ \\
\hline $\begin{array}{l}14 / 7 / 202 \\
0\end{array}$ & $\begin{array}{l}0.96847 \\
6\end{array}$ & $\begin{array}{l}0.03575 \\
3\end{array}$ & 1.37E-05 & $\begin{array}{l}0.00019 \\
5\end{array}$ & $\begin{array}{l}0.40916 \\
9\end{array}$ & $\begin{array}{l}0.00670516 \\
9\end{array}$ & $\begin{array}{l}0.0841220 \\
8\end{array}$ & $\begin{array}{l}0.08412222 \\
7\end{array}$ & $\begin{array}{l}2.16309 \mathrm{E} \\
-14\end{array}$ \\
\hline $\begin{array}{l}15 / 7 / 202 \\
0\end{array}$ & 0.97766 & $\begin{array}{l}0.03617 \\
3\end{array}$ & $1.38 \mathrm{E}-05$ & $\begin{array}{l}0.00019 \\
2\end{array}$ & $\begin{array}{l}0.39577 \\
4\end{array}$ & $\begin{array}{l}0.01400607 \\
8\end{array}$ & 0.09022 & $\begin{array}{l}0.09022000 \\
2\end{array}$ & $\begin{array}{l}4.58107 \mathrm{E} \\
-18\end{array}$ \\
\hline $\begin{array}{l}18 / 7 / 202 \\
0\end{array}$ & $\begin{array}{l}0.97433 \\
3\end{array}$ & $\begin{array}{l}0.03616 \\
5\end{array}$ & $1.83 \mathrm{E}-05$ & $\begin{array}{l}0.00025 \\
6 \\
\end{array}$ & $\begin{array}{l}0.38699 \\
2\end{array}$ & $\begin{array}{l}0.00218259 \\
8\end{array}$ & $\begin{array}{l}0.1108254 \\
4\end{array}$ & $\begin{array}{l}0.11082542 \\
3\end{array}$ & $\begin{array}{l}2.75904 \mathrm{E} \\
-16\end{array}$ \\
\hline $\begin{array}{l}19 / 7 / 202 \\
0\end{array}$ & $\begin{array}{l}0.97277 \\
6 \\
\end{array}$ & $\begin{array}{l}0.03614 \\
8\end{array}$ & $1.56 \mathrm{E}-05$ & $\begin{array}{l}0.00021 \\
8 \\
\end{array}$ & $\begin{array}{l}0.37407 \\
5 \\
\end{array}$ & 0.00742937 & $\begin{array}{l}0.1184948 \\
8\end{array}$ & $\begin{array}{l}0.11849492 \\
3\end{array}$ & $\begin{array}{l}1.82794 \mathrm{E} \\
-15\end{array}$ \\
\hline
\end{tabular}

Table 5: Fitting observed data of females to model

\begin{tabular}{|c|c|c|c|c|c|c|c|c|c|}
\hline DATE & $h_{\mathrm{f}}$ & ]$_{2}$ & if & If & \}$_{2}$ & 国2 & $\begin{array}{l}\text { Observed } \\
\text { Data }\end{array}$ & $\begin{array}{l}\text { Predict } \\
\text { ed }\end{array}$ & $\begin{array}{l}\text { Least } \\
\text { square } \\
\text { error }\end{array}$ \\
\hline $\begin{array}{l}18 / 4 / 20 \\
20\end{array}$ & $\begin{array}{l}0.4887471 \\
24\end{array}$ & $\begin{array}{l}6.19227 \mathrm{E}- \\
05\end{array}$ & 1.67E-07 & $1.67 \mathrm{E}-07$ & $\begin{array}{l}3.35334 \mathrm{E}- \\
05\end{array}$ & 18 & -0.017 & $\begin{array}{l}7.71 E- \\
\end{array}$ & $\begin{array}{l}8.63723 \\
E-05\end{array}$ \\
\hline $\begin{array}{l}20 / 4 / 20 \\
20\end{array}$ & $\begin{array}{l}0.4935339 \\
57\end{array}$ & $\begin{array}{l}6.84923 \mathrm{E}- \\
05\end{array}$ & $\begin{array}{l}7.68676 E- \\
05\end{array}$ & $\begin{array}{l}7.68676 \mathrm{E}- \\
05\end{array}$ & $\begin{array}{l}0.0160810 \\
46\end{array}$ & $\begin{array}{l}0.0159936 \\
78\end{array}$ & $\begin{array}{l}- \\
0.007108 \\
32\end{array}$ & $\begin{array}{l}-6.79 \mathrm{E}- \\
03\end{array}$ & $\begin{array}{l}1.02603 \\
E-07\end{array}$ \\
\hline $\begin{array}{l}21 / 4 / 20 \\
20\end{array}$ & $\begin{array}{l}0.4930546 \\
06\end{array}$ & $\begin{array}{l}6.52633 E- \\
05\end{array}$ & $\begin{array}{l}0.0003047 \\
72\end{array}$ & $\begin{array}{l}0.0003047 \\
72\end{array}$ & $\begin{array}{l}0.0314764 \\
05\end{array}$ & $\begin{array}{l}0.0313375 \\
93\end{array}$ & $\begin{array}{l}- \\
0.002535 \\
08\end{array}$ & $\begin{array}{l}-3.11 \mathrm{E}- \\
03\end{array}$ & $\begin{array}{l}3.26046 \\
E-07\end{array}$ \\
\hline $\begin{array}{l}22 / 4 / 20 \\
20\end{array}$ & $\begin{array}{l}0.4666839 \\
87\end{array}$ & $\begin{array}{l}0.0001002 \\
12\end{array}$ & $\begin{array}{l}6.60128 \mathrm{E}- \\
06\end{array}$ & $\begin{array}{l}6.60128 \mathrm{E}- \\
06\end{array}$ & 0 & $\begin{array}{l}0.0061198 \\
76\end{array}$ & $\begin{array}{l}.001797 \\
4\end{array}$ & $\begin{array}{l}.82 \mathrm{E}- \\
3\end{array}$ & $\begin{array}{l}6.67794 \\
E-10\end{array}$ \\
\hline $\begin{array}{l}23 / 4 / 20 \\
20\end{array}$ & $\begin{array}{l}0.4808631 \\
61\end{array}$ & $\begin{array}{l}8.37416 \mathrm{E}- \\
05\end{array}$ & $\begin{array}{l}0.0005734 \\
09\end{array}$ & $\begin{array}{l}0.0005734 \\
09\end{array}$ & $\begin{array}{l}0.0692380 \\
67\end{array}$ & $\begin{array}{l}0.0690451 \\
91\end{array}$ & 0.005895 & $\begin{array}{l}5.86 \mathrm{E}- \\
03\end{array}$ & $\begin{array}{l}1.23843 \\
E-09\end{array}$ \\
\hline $\begin{array}{l}24 / 4 / 20 \\
20\end{array}$ & $\begin{array}{l}0.4127602 \\
63\end{array}$ & $\begin{array}{l}2.43057 \mathrm{E}- \\
05\end{array}$ & $\begin{array}{l}0.0001509 \\
02\end{array}$ & $\begin{array}{l}0.0001509 \\
02\end{array}$ & 0 & 0 & $\begin{array}{l}0.009763 \\
36\end{array}$ & $\begin{array}{l}9.79 \mathrm{E}- \\
03\end{array}$ & $\begin{array}{l}5.46455 \\
E-10\end{array}$ \\
\hline $\begin{array}{l}29 / 4 / 20 \\
20\end{array}$ & $\begin{array}{l}0.4993276 \\
45\end{array}$ & $\begin{array}{l}0.0004528 \\
35\end{array}$ & $\begin{array}{l}0.0001759 \\
19\end{array}$ & $1.76 \mathrm{E}-04$ & $\begin{array}{l}0.3967536 \\
87\end{array}$ & $\begin{array}{l}0.0558782 \\
02\end{array}$ & $\begin{array}{l}0.025868 \\
76\end{array}$ & $\begin{array}{l}2.58 \mathrm{E}- \\
02\end{array}$ & $\begin{array}{l}9.59804 \\
E-10\end{array}$ \\
\hline
\end{tabular}

This publication is licensed under Creative Commons Attribution CC BY. 


\begin{tabular}{|c|c|c|c|c|c|c|c|c|c|}
\hline $\begin{array}{l}30 / 4 / 20 \\
20\end{array}$ & $\begin{array}{l}0.5015619 \\
76\end{array}$ & $\begin{array}{l}0.0001656 \\
94\end{array}$ & $\begin{array}{l}0.0003587 \\
76\end{array}$ & $\begin{array}{l}0.0003617 \\
25\end{array}$ & $\begin{array}{l}0.3705281 \\
3\end{array}$ & $\begin{array}{l}0.0788238 \\
83\end{array}$ & $\begin{array}{l}0.028482 \\
88\end{array}$ & $\begin{array}{l}2.85 \mathrm{E}- \\
02\end{array}$ & $\begin{array}{l}2.25429 \\
E-11\end{array}$ \\
\hline $\begin{array}{l}2 / 5 / 202 \\
0\end{array}$ & $\begin{array}{l}0.4958592 \\
89\end{array}$ & $\begin{array}{l}0.0001590 \\
02\end{array}$ & $5.74 \mathrm{E}-04$ & $2.92 \mathrm{E}-03$ & $\begin{array}{l}0.3469651 \\
65\end{array}$ & $\begin{array}{l}0.0972104 \\
32\end{array}$ & $\begin{array}{l}0.033150 \\
24\end{array}$ & $\begin{array}{l}2.98 \mathrm{E}- \\
02\end{array}$ & $\begin{array}{l}1.13755 \\
E-05\end{array}$ \\
\hline $\begin{array}{l}3 / 5 / 202 \\
0\end{array}$ & $\begin{array}{l}0.5169563 \\
81\end{array}$ & $\begin{array}{l}0.0002260 \\
03\end{array}$ & $\begin{array}{l}0.0006157 \\
98\end{array}$ & $\begin{array}{l}0.0006157 \\
98\end{array}$ & $\begin{array}{l}0.4061295 \\
97\end{array}$ & $\begin{array}{l}0.0361107 \\
51\end{array}$ & 0.035215 & $\begin{array}{l}3.52 \mathrm{E}- \\
02\end{array}$ & $\begin{array}{l}4.43003 \\
E-13\end{array}$ \\
\hline $\begin{array}{l}5 / 5 / 202 \\
0\end{array}$ & $\begin{array}{l}0.5686085 \\
37\end{array}$ & $\begin{array}{l}0.0002268 \\
43\end{array}$ & $\begin{array}{l}0.0013139 \\
88\end{array}$ & $\begin{array}{l}0.0013139 \\
88\end{array}$ & $\begin{array}{l}0.1023557 \\
92\end{array}$ & $\begin{array}{l}0.3357318 \\
32\end{array}$ & $\begin{array}{l}0.038835 \\
48\end{array}$ & $\begin{array}{l}3.91 \mathrm{E}- \\
02\end{array}$ & $\begin{array}{l}7.79429 \\
E-08\end{array}$ \\
\hline $\begin{array}{l}6 / 5 / 202 \\
0\end{array}$ & $\begin{array}{l}0.7367717 \\
13\end{array}$ & $\begin{array}{l}0.0002785 \\
7\end{array}$ & $\begin{array}{l}0.0011412 \\
34\end{array}$ & $\begin{array}{l}0.0011412 \\
34\end{array}$ & $\begin{array}{l}0.3962440 \\
87\end{array}$ & $\begin{array}{l}0.0406587 \\
81\end{array}$ & $\begin{array}{l}0.040402 \\
72\end{array}$ & $\begin{array}{l}4.03 \mathrm{E}- \\
02\end{array}$ & $\begin{array}{l}2.04333 \\
\mathrm{E}-08\end{array}$ \\
\hline $\begin{array}{l}7 / 5 / 202 \\
0\end{array}$ & $\begin{array}{l}0.7916368 \\
19\end{array}$ & $\begin{array}{l}0.0002864 \\
66\end{array}$ & 0.0002778 & 0.0002778 & $\begin{array}{l}0.3400257 \\
97\end{array}$ & $\begin{array}{l}0.0960040 \\
54\end{array}$ & $\begin{array}{l}0.041815 \\
64\end{array}$ & $\begin{array}{l}4.17 \mathrm{E}- \\
02\end{array}$ & $\begin{array}{l}5.04011 \\
\mathrm{E}-09\end{array}$ \\
\hline $\begin{array}{l}8 / 5 / 202 \\
0\end{array}$ & $\begin{array}{l}0.5460827 \\
45\end{array}$ & $\begin{array}{l}0.0003805 \\
72\end{array}$ & $\begin{array}{l}0.0002347 \\
35\end{array}$ & $\begin{array}{l}0.0002835 \\
82\end{array}$ & $\begin{array}{l}0.1865081 \\
69\end{array}$ & $\begin{array}{l}0.2482340 \\
89\end{array}$ & 0.04308 & $\begin{array}{l}4.31 \mathrm{E}- \\
02\end{array}$ & $\begin{array}{l}3.76001 \\
E-14\end{array}$ \\
\hline $\begin{array}{l}9 / 5 / 202 \\
0\end{array}$ & $\begin{array}{l}0.5098859 \\
11\end{array}$ & $\begin{array}{l}0.0003355 \\
08\end{array}$ & $\begin{array}{l}0.0006373 \\
38\end{array}$ & $\begin{array}{l}0.0006681 \\
23\end{array}$ & $\begin{array}{l}0.4036882 \\
39\end{array}$ & $\begin{array}{l}0.0304360 \\
07\end{array}$ & $\begin{array}{l}0.044201 \\
56\end{array}$ & $\begin{array}{l}4.42 \mathrm{E}- \\
02\end{array}$ & $\begin{array}{l}5.88061 \\
\mathrm{E}-15\end{array}$ \\
\hline $\begin{array}{l}10 / 5 / 20 \\
20\end{array}$ & $\begin{array}{l}0.7308048 \\
35\end{array}$ & $\begin{array}{l}0.0003512 \\
69\end{array}$ & $\begin{array}{l}0.0002601 \\
53\end{array}$ & $\begin{array}{l}0.0006803 \\
9\end{array}$ & $\begin{array}{l}0.3638141 \\
24\end{array}$ & $\begin{array}{l}0.0695224 \\
95\end{array}$ & $\begin{array}{l}0.045186 \\
08\end{array}$ & $\begin{array}{l}4.52 \mathrm{E}- \\
02\end{array}$ & $\begin{array}{l}1.28393 \\
\mathrm{E}-14\end{array}$ \\
\hline $\begin{array}{l}12 / 5 / 20 \\
20\end{array}$ & $\begin{array}{l}0.6815636 \\
31\end{array}$ & $\begin{array}{l}0.0003430 \\
97\end{array}$ & $\begin{array}{l}0.0001582 \\
51\end{array}$ & $\begin{array}{l}0.0002941 \\
58\end{array}$ & $\begin{array}{l}0.3192852 \\
79\end{array}$ & $\begin{array}{l}0.0473674 \\
01\end{array}$ & $\begin{array}{l}0.046767 \\
04\end{array}$ & $\begin{array}{l}4.68 \mathrm{E}- \\
02\end{array}$ & $\begin{array}{l}3.97701 \\
E-14\end{array}$ \\
\hline $\begin{array}{l}15 / 5 / 20 \\
20\end{array}$ & $\begin{array}{l}0.7877886 \\
94\end{array}$ & $\begin{array}{l}0.0003568 \\
09\end{array}$ & $\begin{array}{l}4.12692 \mathrm{E}- \\
05\end{array}$ & $\begin{array}{l}7.29187 \mathrm{E}- \\
05\end{array}$ & $\begin{array}{l}0.0605687 \\
26\end{array}$ & $\begin{array}{l}0.0046300 \\
34\end{array}$ & $\begin{array}{l}0.048254 \\
68 \\
\end{array}$ & $\begin{array}{l}4.83 \mathrm{E}- \\
02\end{array}$ & $\begin{array}{l}5.25445 \\
\mathrm{E}-14 \\
\end{array}$ \\
\hline $\begin{array}{l}18 / 5 / 20 \\
20\end{array}$ & $\begin{array}{l}0.8919059 \\
46\end{array}$ & $\begin{array}{l}0.0003794 \\
67\end{array}$ & $\begin{array}{l}0.0001072 \\
63\end{array}$ & $\begin{array}{l}0.0001072 \\
63\end{array}$ & $\begin{array}{l}0.0291396 \\
44\end{array}$ & $\begin{array}{l}0.4107719 \\
12\end{array}$ & 0.04882 & $\begin{array}{l}4.88 \mathrm{E}- \\
02\end{array}$ & $\begin{array}{l}6.30928 \\
E-13\end{array}$ \\
\hline $\begin{array}{l}20 / 5 / 20 \\
20\end{array}$ & $\begin{array}{l}0.8083569 \\
92\end{array}$ & $\begin{array}{l}0.0003047 \\
59\end{array}$ & $\begin{array}{l}0.0001806 \\
53\end{array}$ & $\begin{array}{l}0.0001806 \\
53\end{array}$ & $\begin{array}{l}0.0087234 \\
47\end{array}$ & 0 & $\begin{array}{l}0.048761 \\
28\end{array}$ & $\begin{array}{l}4.88 \mathrm{E}- \\
02\end{array}$ & $\begin{array}{l}9.93749 \\
E-16\end{array}$ \\
\hline $\begin{array}{l}26 / 5 / 20 \\
20\end{array}$ & $\begin{array}{l}0.8032731 \\
16\end{array}$ & $\begin{array}{l}0.0004141 \\
38\end{array}$ & $\begin{array}{l}8.56216 \mathrm{E}- \\
05\end{array}$ & $\begin{array}{l}8.56216 \mathrm{E}- \\
05\end{array}$ & $\begin{array}{l}0.0029456 \\
95\end{array}$ & $\begin{array}{l}0.0001439 \\
37\end{array}$ & $\begin{array}{l}0.047001 \\
12\end{array}$ & $\begin{array}{l}4.70 \mathrm{E}- \\
02\end{array}$ & $\begin{array}{l}1.37459 \\
\mathrm{E}-15\end{array}$ \\
\hline $\begin{array}{l}27 / 5 / 20 \\
20\end{array}$ & $\begin{array}{l}0.7362210 \\
54\end{array}$ & $\begin{array}{l}0.0004438 \\
46\end{array}$ & $\begin{array}{l}0.0002158 \\
39\end{array}$ & $\begin{array}{l}0.0002158 \\
39\end{array}$ & $\begin{array}{l}0.0022935 \\
23\end{array}$ & $\begin{array}{l}0.0022935 \\
23\end{array}$ & $\begin{array}{l}0.046537 \\
24\end{array}$ & $\begin{array}{l}4.65 \mathrm{E}- \\
02\end{array}$ & $\begin{array}{l}3.06792 \\
E-16\end{array}$ \\
\hline $\begin{array}{l}28 / 5 / 20 \\
20\end{array}$ & $\begin{array}{l}0.6812299 \\
46\end{array}$ & $\begin{array}{l}0.0003803 \\
07\end{array}$ & $\begin{array}{l}0.0003680 \\
78\end{array}$ & $\begin{array}{l}0.0003680 \\
78\end{array}$ & $\begin{array}{l}0.0081147 \\
35\end{array}$ & $\begin{array}{l}0.0018726 \\
55\end{array}$ & 0.04604 & $\begin{array}{l}4.60 \mathrm{E}- \\
02\end{array}$ & $\begin{array}{l}3.0525 \mathrm{E} \\
-18 \\
\end{array}$ \\
\hline $\begin{array}{l}30 / 5 / 20 \\
20\end{array}$ & $\begin{array}{l}0.5852956 \\
44\end{array}$ & $\begin{array}{l}0.0003477 \\
68\end{array}$ & $\begin{array}{l}0.0002371 \\
93\end{array}$ & $\begin{array}{l}0.0002371 \\
93\end{array}$ & $\begin{array}{l}0.0139831 \\
58\end{array}$ & $\begin{array}{l}0.0005299 \\
75\end{array}$ & $\begin{array}{l}0.045515 \\
16\end{array}$ & $\begin{array}{l}4.55 \mathrm{E}- \\
02\end{array}$ & $\begin{array}{l}6.18921 \\
\text { E-16 }\end{array}$ \\
\hline $\begin{array}{l}31 / 5 / 20 \\
20\end{array}$ & $\begin{array}{l}0.5500580 \\
8\end{array}$ & $\begin{array}{l}0.0003826 \\
27\end{array}$ & $\begin{array}{l}0.0001083 \\
66\end{array}$ & $\begin{array}{l}0.0001083 \\
66\end{array}$ & $\begin{array}{l}0.0069475 \\
15\end{array}$ & $\begin{array}{l}0.0004915 \\
22\end{array}$ & $\begin{array}{l}0.044968 \\
48\end{array}$ & $\begin{array}{l}4.50 \mathrm{E}- \\
02\end{array}$ & $\begin{array}{l}3.05777 \\
\text { E-16 }\end{array}$ \\
\hline $\begin{array}{l}1 / 6 / 202 \\
0\end{array}$ & $\begin{array}{l}0.5944578 \\
73\end{array}$ & $\begin{array}{l}0.0004937 \\
4\end{array}$ & $\begin{array}{l}0.0001020 \\
5\end{array}$ & $\begin{array}{l}0.0001020 \\
5\end{array}$ & $\begin{array}{l}0.0036968 \\
71\end{array}$ & $\begin{array}{l}0.0023145 \\
59\end{array}$ & $\begin{array}{l}0.044405 \\
72\end{array}$ & $\begin{array}{l}4.44 \mathrm{E}- \\
02\end{array}$ & $\begin{array}{l}2.55774 \\
E-17\end{array}$ \\
\hline $\begin{array}{l}2 / 6 / 202 \\
0\end{array}$ & $\begin{array}{l}0.6810608 \\
21\end{array}$ & $\begin{array}{l}0.0003753 \\
46\end{array}$ & $\begin{array}{l}0.0001271 \\
62\end{array}$ & $\begin{array}{l}0.0001271 \\
62\end{array}$ & $\begin{array}{l}0.0073976 \\
97\end{array}$ & $\begin{array}{l}0.0008703 \\
22\end{array}$ & $\begin{array}{l}0.043832 \\
64\end{array}$ & $\begin{array}{l}4.38 \mathrm{E}- \\
02\end{array}$ & $\begin{array}{l}4.7555 \mathrm{E} \\
-17 \\
\end{array}$ \\
\hline $\begin{array}{l}3 / 6 / 202 \\
0\end{array}$ & $\begin{array}{l}0.8718734 \\
86\end{array}$ & $\begin{array}{l}0.0006934 \\
38\end{array}$ & $\begin{array}{l}0.0001098 \\
83\end{array}$ & $\begin{array}{l}0.0001098 \\
83\end{array}$ & 0 & $\begin{array}{l}0.0121951 \\
22\end{array}$ & 0.043255 & $\begin{array}{l}4.33 \mathrm{E}- \\
02\end{array}$ & $\begin{array}{l}4.62965 \\
E-17\end{array}$ \\
\hline $\begin{array}{l}4 / 6 / 202 \\
0\end{array}$ & $\begin{array}{l}0.9298929 \\
07\end{array}$ & $\begin{array}{l}0.0007550 \\
96\end{array}$ & $\begin{array}{l}0.0002890 \\
2\end{array}$ & $\begin{array}{l}0.0002890 \\
2\end{array}$ & $\begin{array}{l}0.1572580 \\
65\end{array}$ & $\begin{array}{l}0.0184346 \\
72\end{array}$ & $\begin{array}{l}0.042678 \\
56\end{array}$ & $\begin{array}{l}4.27 \mathrm{E}- \\
02\end{array}$ & $\begin{array}{l}4.26227 \\
\mathrm{E}-17 \\
\end{array}$ \\
\hline $\begin{array}{l}5 / 6 / 202 \\
0\end{array}$ & $\begin{array}{l}0.5713502 \\
21\end{array}$ & $\begin{array}{l}0.0010606 \\
08\end{array}$ & $\begin{array}{l}0.0003321 \\
57\end{array}$ & $\begin{array}{l}0.0003321 \\
57\end{array}$ & $\begin{array}{l}0.1902985 \\
07\end{array}$ & $\begin{array}{l}0.0025955 \\
99\end{array}$ & $\begin{array}{l}0.042109 \\
08\end{array}$ & $\begin{array}{l}4.21 \mathrm{E}- \\
02\end{array}$ & $\begin{array}{l}7.34556 \\
E-17\end{array}$ \\
\hline $\begin{array}{l}6 / 6 / 202 \\
0\end{array}$ & $\begin{array}{l}0.4923947 \\
27\end{array}$ & $\begin{array}{l}0.0010758 \\
2\end{array}$ & $\begin{array}{l}0.0003259 \\
22\end{array}$ & $\begin{array}{l}0.0003259 \\
22\end{array}$ & 0.25 & $\begin{array}{l}0.0091744 \\
53\end{array}$ & $\begin{array}{l}0.041552 \\
32\end{array}$ & $\begin{array}{l}4.16 \mathrm{E}- \\
02\end{array}$ & $\begin{array}{l}2.75308 \\
E-17\end{array}$ \\
\hline $\begin{array}{l}7 / 6 / 202 \\
0\end{array}$ & $\begin{array}{l}0.5099478 \\
25\end{array}$ & $\begin{array}{l}0.0012642 \\
34\end{array}$ & $\begin{array}{l}0.0002492 \\
5\end{array}$ & $\begin{array}{l}0.0002492 \\
5\end{array}$ & $\begin{array}{l}0.1377245 \\
51\end{array}$ & $\begin{array}{l}0.0014345 \\
66\end{array}$ & $\begin{array}{l}0.041014 \\
04\end{array}$ & $\begin{array}{l}4.10 \mathrm{E}- \\
02\end{array}$ & $\begin{array}{l}3.05794 \\
E-17\end{array}$ \\
\hline $\begin{array}{l}9 / 6 / 202 \\
0\end{array}$ & $\begin{array}{l}0.6218546 \\
24\end{array}$ & $\begin{array}{l}0.0013500 \\
12\end{array}$ & $\begin{array}{l}0.0001507 \\
24\end{array}$ & $\begin{array}{l}0.0001507 \\
24\end{array}$ & $\begin{array}{l}0.0944881 \\
89\end{array}$ & $\begin{array}{l}0.0130871 \\
13\end{array}$ & 0.0405 & $\begin{array}{l}4.05 \mathrm{E}- \\
02\end{array}$ & $\begin{array}{l}2.17046 \\
E-16\end{array}$ \\
\hline $\begin{array}{l}10 / 6 / 20 \\
20\end{array}$ & $\begin{array}{l}0.8209434 \\
7\end{array}$ & $\begin{array}{l}0.0013044 \\
44\end{array}$ & $\begin{array}{l}0.0004057 \\
87\end{array}$ & $\begin{array}{l}0.0004057 \\
87\end{array}$ & $\begin{array}{l}0.4139707 \\
35\end{array}$ & 0 & $\begin{array}{l}0.040015 \\
96\end{array}$ & $\begin{array}{l}4.00 E- \\
02\end{array}$ & $\begin{array}{l}9.43201 \\
\mathrm{E}-15\end{array}$ \\
\hline
\end{tabular}

This publication is licensed under Creative Commons Attribution CC BY. 


\begin{tabular}{|c|c|c|c|c|c|c|c|c|c|}
\hline $\begin{array}{l}11 / 6 / 20 \\
20\end{array}$ & $\begin{array}{l}0.9997649 \\
9\end{array}$ & $\begin{array}{l}0.0012390 \\
45\end{array}$ & $\begin{array}{l}0.0002167 \\
48\end{array}$ & $\begin{array}{l}0.0002167 \\
48\end{array}$ & $\begin{array}{l}0.1818181 \\
82\end{array}$ & 0.0010071 & $\begin{array}{l}0.039567 \\
68\end{array}$ & $\begin{array}{l}3.96 \mathrm{E}- \\
02\end{array}$ & $\begin{array}{l}4.51484 \\
E-15\end{array}$ \\
\hline $\begin{array}{l}12 / 6 / 20 \\
20\end{array}$ & $\begin{array}{l}0.9992882 \\
38\end{array}$ & $\begin{array}{l}0.0012626 \\
05\end{array}$ & $\begin{array}{l}0.0004063 \\
93\end{array}$ & $\begin{array}{l}0.0004063 \\
93\end{array}$ & 0.4 & $\begin{array}{l}0.0009209 \\
01\end{array}$ & $\begin{array}{l}0.039160 \\
92\end{array}$ & $\begin{array}{l}3.92 \mathrm{E}- \\
02\end{array}$ & $\begin{array}{l}1.75019 \\
E-20\end{array}$ \\
\hline $\begin{array}{l}13 / 6 / 20 \\
20\end{array}$ & $\begin{array}{l}0.9960790 \\
63\end{array}$ & $\begin{array}{l}0.0012886 \\
48\end{array}$ & $\begin{array}{l}0.0002656 \\
05\end{array}$ & $\begin{array}{l}0.0002656 \\
05\end{array}$ & 0.1875 & $\begin{array}{l}0.0020194 \\
63\end{array}$ & $\begin{array}{l}0.038801 \\
44 \\
\end{array}$ & $\begin{array}{l}3.88 \mathrm{E}- \\
02\end{array}$ & $\begin{array}{l}1.29659 \\
\text { E-16 }\end{array}$ \\
\hline $\begin{array}{l}15 / 6 / 20 \\
20\end{array}$ & $\begin{array}{l}0.9928682 \\
9\end{array}$ & 0.0012879 & $\begin{array}{l}0.0002102 \\
33\end{array}$ & $\begin{array}{l}0.0002102 \\
33\end{array}$ & $\begin{array}{l}0.1240601 \\
5\end{array}$ & 0 & 0.038495 & $\begin{array}{l}3.85 \mathrm{E}- \\
02\end{array}$ & $\begin{array}{l}8.85949 \\
E-17\end{array}$ \\
\hline $\begin{array}{l}16 / 6 / 20 \\
20\end{array}$ & $\begin{array}{l}0.9963332 \\
59\end{array}$ & $\begin{array}{l}0.0012878 \\
28\end{array}$ & $\begin{array}{l}0.0001541 \\
06\end{array}$ & $\begin{array}{l}0.0001541 \\
06\end{array}$ & $\begin{array}{l}0.1503759 \\
4\end{array}$ & $\begin{array}{l}0.0020175 \\
14\end{array}$ & $\begin{array}{l}0.038247 \\
36\end{array}$ & $\begin{array}{l}3.82 \mathrm{E}- \\
02\end{array}$ & $\begin{array}{l}6.7164 \mathrm{E} \\
-16\end{array}$ \\
\hline $\begin{array}{l}17 / 6 / 20 \\
20\end{array}$ & $\begin{array}{l}0.9948881 \\
08\end{array}$ & $\begin{array}{l}0.0013304 \\
98\end{array}$ & $\begin{array}{l}0.0002020 \\
35\end{array}$ & $\begin{array}{l}0.0002020 \\
35\end{array}$ & $\begin{array}{l}0.0733695 \\
65\end{array}$ & $\begin{array}{l}0.0059358 \\
94\end{array}$ & $\begin{array}{l}0.038064 \\
28 \\
\end{array}$ & $\begin{array}{l}3.81 \mathrm{E}- \\
02\end{array}$ & $\begin{array}{l}7.1194 \mathrm{E} \\
-17 \\
\end{array}$ \\
\hline $\begin{array}{l}18 / 6 / 20 \\
20\end{array}$ & $\begin{array}{l}0.9940645 \\
94\end{array}$ & $\begin{array}{l}0.0013036 \\
2\end{array}$ & $\begin{array}{l}0.0010083 \\
48\end{array}$ & $\begin{array}{l}0.0010083 \\
48\end{array}$ & $\begin{array}{l}0.4459644 \\
78\end{array}$ & $\begin{array}{l}0.0252359 \\
1\end{array}$ & $\begin{array}{l}0.037951 \\
52\end{array}$ & $\begin{array}{l}3.80 \mathrm{E}- \\
02\end{array}$ & $\begin{array}{l}1.53347 \\
E-15\end{array}$ \\
\hline $\begin{array}{l}19 / 6 / 20 \\
20\end{array}$ & $\begin{array}{l}0.6743964 \\
34\end{array}$ & $\begin{array}{l}0.0017224 \\
37\end{array}$ & $\begin{array}{l}0.0004189 \\
32\end{array}$ & $\begin{array}{l}0.0004189 \\
32\end{array}$ & $\begin{array}{l}0.4631685 \\
18\end{array}$ & $\begin{array}{l}0.0085829 \\
68\end{array}$ & $\begin{array}{l}0.037914 \\
84\end{array}$ & $\begin{array}{l}3.79 \mathrm{E}- \\
02\end{array}$ & $\begin{array}{l}1.49504 \\
E-15\end{array}$ \\
\hline $\begin{array}{l}20 / 6 / 20 \\
20\end{array}$ & 0.8003572 & $\begin{array}{l}0.0016960 \\
47\end{array}$ & $\begin{array}{l}0.0003506 \\
87\end{array}$ & $\begin{array}{l}0.0003506 \\
87\end{array}$ & $\begin{array}{l}0.4616591 \\
07\end{array}$ & $\begin{array}{l}0.0105061 \\
46\end{array}$ & 0.03796 & $\begin{array}{l}3.80 \mathrm{E}- \\
02\end{array}$ & $\begin{array}{l}2.27303 \\
E-14\end{array}$ \\
\hline $\begin{array}{l}21 / 6 / 20 \\
20\end{array}$ & $\begin{array}{l}0.9946279 \\
54\end{array}$ & $\begin{array}{l}0.0016587 \\
91\end{array}$ & $\begin{array}{l}0.0010764 \\
98\end{array}$ & $\begin{array}{l}0.0010764 \\
98\end{array}$ & $\begin{array}{l}0.4647207 \\
13\end{array}$ & $\begin{array}{l}0.0076950 \\
2\end{array}$ & $\begin{array}{l}0.038092 \\
76\end{array}$ & $\begin{array}{l}3.81 \mathrm{E}- \\
02\end{array}$ & $\begin{array}{l}1.52796 \\
E-15\end{array}$ \\
\hline $\begin{array}{l}22 / 6 / 20 \\
20\end{array}$ & $\begin{array}{l}0.9936181 \\
22 \\
\end{array}$ & 0.0016845 & $\begin{array}{l}0.0001515 \\
5 \\
\end{array}$ & $\begin{array}{l}0.0001515 \\
5 \\
\end{array}$ & $\begin{array}{l}0.4557377 \\
85\end{array}$ & $\begin{array}{l}0.0168272 \\
61 \\
\end{array}$ & $\begin{array}{l}0.038318 \\
88 \\
\end{array}$ & $\begin{array}{l}3.83 \mathrm{E}- \\
02\end{array}$ & $\begin{array}{l}1.658 \mathrm{E}- \\
14\end{array}$ \\
\hline $\begin{array}{l}23 / 6 / 20 \\
20\end{array}$ & $\begin{array}{l}0.9946014 \\
95\end{array}$ & $\begin{array}{l}0.0017129 \\
3\end{array}$ & $\begin{array}{l}0.0004348 \\
46\end{array}$ & $\begin{array}{l}0.0004348 \\
46\end{array}$ & $\begin{array}{l}0.4626770 \\
86\end{array}$ & $\begin{array}{l}0.0097931 \\
23\end{array}$ & $\begin{array}{l}0.038644 \\
12\end{array}$ & $\begin{array}{l}3.86 \mathrm{E}- \\
02\end{array}$ & $\begin{array}{l}1.54792 \\
\text { E-09 }\end{array}$ \\
\hline $\begin{array}{l}24 / 6 / 20 \\
20\end{array}$ & $\begin{array}{l}0.9927621 \\
59\end{array}$ & $\begin{array}{l}0.0017287 \\
84\end{array}$ & $\begin{array}{l}0.0008375 \\
22\end{array}$ & $\begin{array}{l}0.0008375 \\
22\end{array}$ & $\begin{array}{l}0.4673717 \\
69\end{array}$ & $\begin{array}{l}0.0048570 \\
98\end{array}$ & $\begin{array}{l}0.039074 \\
24\end{array}$ & $\begin{array}{l}3.91 \mathrm{E}- \\
02\end{array}$ & $\begin{array}{l}9.99161 \\
E-10\end{array}$ \\
\hline $\begin{array}{l}25 / 6 / 20 \\
20\end{array}$ & $\begin{array}{l}0.9931111 \\
57\end{array}$ & $\begin{array}{l}0.0017815 \\
57\end{array}$ & $\begin{array}{l}0.0006574 \\
05\end{array}$ & $\begin{array}{l}0.0006574 \\
05\end{array}$ & $\begin{array}{l}0.4648942 \\
91\end{array}$ & $\begin{array}{l}0.0068961 \\
63\end{array}$ & 0.039615 & $\begin{array}{l}3.96 \mathrm{E}- \\
02\end{array}$ & $\begin{array}{l}4.34195 \\
E-10\end{array}$ \\
\hline $\begin{array}{l}26 / 6 / 20 \\
20\end{array}$ & $\begin{array}{l}0.9921943 \\
31\end{array}$ & $\begin{array}{l}0.0018039 \\
21\end{array}$ & $\begin{array}{l}0.0006493 \\
38\end{array}$ & $\begin{array}{l}0.0006493 \\
38\end{array}$ & $\begin{array}{l}0.4512687 \\
59\end{array}$ & $\begin{array}{l}0.0199275 \\
56\end{array}$ & $\begin{array}{l}0.040272 \\
16\end{array}$ & $\begin{array}{l}4.03 E- \\
02\end{array}$ & $\begin{array}{l}3.49016 \\
E-10\end{array}$ \\
\hline $\begin{array}{l}27 / 6 / 20 \\
20\end{array}$ & $\begin{array}{l}0.9738889 \\
28\end{array}$ & $\begin{array}{l}0.0018157 \\
27\end{array}$ & $\begin{array}{l}0.0011373 \\
02\end{array}$ & $\begin{array}{l}0.0011373 \\
02\end{array}$ & $\begin{array}{l}0.4565013 \\
26\end{array}$ & $\begin{array}{l}0.0138664 \\
33\end{array}$ & $\begin{array}{l}0.041051 \\
48\end{array}$ & $\begin{array}{l}4.09 \mathrm{E}- \\
02\end{array}$ & $\begin{array}{l}2.91447 \\
E-08\end{array}$ \\
\hline $\begin{array}{l}28 / 6 / 20 \\
20\end{array}$ & $\begin{array}{l}0.9756237 \\
37\end{array}$ & $\begin{array}{l}0.0018035 \\
01\end{array}$ & $\begin{array}{l}0.0011806 \\
25\end{array}$ & $\begin{array}{l}0.0011806 \\
25\end{array}$ & $\begin{array}{l}0.4642001 \\
28\end{array}$ & $\begin{array}{l}0.0051561 \\
42\end{array}$ & $\begin{array}{l}0.041958 \\
72\end{array}$ & $\begin{array}{l}4.20 \mathrm{E}- \\
02\end{array}$ & $\begin{array}{l}4.18487 \\
E-10\end{array}$ \\
\hline $\begin{array}{l}1 / 7 / 202 \\
0\end{array}$ & $\begin{array}{l}0.9736289 \\
23\end{array}$ & $\begin{array}{l}0.0018335 \\
91\end{array}$ & $\begin{array}{l}0.0010663 \\
46\end{array}$ & $\begin{array}{l}0.0010663 \\
46\end{array}$ & $\begin{array}{l}0.4629866 \\
2\end{array}$ & $\begin{array}{l}0.0017812 \\
88\end{array}$ & $\begin{array}{l}0.045505 \\
56\end{array}$ & $\begin{array}{l}4.56 \mathrm{E}- \\
02\end{array}$ & $\begin{array}{l}1.05461 \\
\text { E-08 }\end{array}$ \\
\hline $\begin{array}{l}2 / 7 / 202 \\
0\end{array}$ & $\begin{array}{l}0.5477244 \\
65\end{array}$ & $\begin{array}{l}0.0026473 \\
51\end{array}$ & $\begin{array}{l}0.0008660 \\
54\end{array}$ & $\begin{array}{l}0.0008660 \\
54\end{array}$ & $\begin{array}{l}0.4479814 \\
94\end{array}$ & $\begin{array}{l}0.0148371 \\
15\end{array}$ & $\begin{array}{l}0.046982 \\
08\end{array}$ & $\begin{array}{l}4.70 \mathrm{E}- \\
02\end{array}$ & $\begin{array}{l}1.28978 \\
E-10\end{array}$ \\
\hline $\begin{array}{l}5 / 7 / 202 \\
0\end{array}$ & $\begin{array}{l}0.5213481 \\
72\end{array}$ & $\begin{array}{l}0.0026129 \\
41\end{array}$ & $\begin{array}{l}0.0007442 \\
16 \\
\end{array}$ & $\begin{array}{l}0.0007442 \\
16 \\
\end{array}$ & $\begin{array}{l}0.4534811 \\
43\end{array}$ & $\begin{array}{l}0.0017607 \\
47 \\
\end{array}$ & 0.052375 & $\begin{array}{l}5.24 \mathrm{E}- \\
02\end{array}$ & $\begin{array}{l}4.50233 \\
\mathrm{E}-10 \\
\end{array}$ \\
\hline $\begin{array}{l}7 / 7 / 202 \\
0\end{array}$ & $\begin{array}{l}0.8153028 \\
61\end{array}$ & $\begin{array}{l}0.0025645 \\
37\end{array}$ & $\begin{array}{l}0.0005088 \\
77\end{array}$ & $\begin{array}{l}0.0005088 \\
77\end{array}$ & $\begin{array}{l}0.4402626 \\
6\end{array}$ & $\begin{array}{l}0.0084127 \\
38\end{array}$ & $\begin{array}{l}0.056830 \\
68\end{array}$ & $\begin{array}{l}5.68 \mathrm{E}- \\
02\end{array}$ & $\begin{array}{l}4.93642 \\
E-10\end{array}$ \\
\hline $\begin{array}{l}9 / 7 / 202 \\
0\end{array}$ & $\begin{array}{l}0.9994291 \\
87\end{array}$ & $\begin{array}{l}0.0342545 \\
13\end{array}$ & $\begin{array}{l}3.71755 \mathrm{E}- \\
05\end{array}$ & $\begin{array}{l}0.0001021 \\
49\end{array}$ & $\begin{array}{l}0.4350616 \\
03\end{array}$ & $\begin{array}{l}0.0058941 \\
06\end{array}$ & $\begin{array}{l}0.062028 \\
44\end{array}$ & $\begin{array}{l}6.20 \mathrm{E}- \\
02\end{array}$ & $\begin{array}{l}5.36191 \\
E-15\end{array}$ \\
\hline $\begin{array}{l}10 / 7 / 20 \\
20\end{array}$ & 1 & $\begin{array}{l}0.0340648 \\
83\end{array}$ & $\begin{array}{l}4.1305 E- \\
05\end{array}$ & $\begin{array}{l}9.13224 \mathrm{E}- \\
05\end{array}$ & $\begin{array}{l}0.4242010 \\
48\end{array}$ & $\begin{array}{l}0.0122685 \\
9\end{array}$ & 0.06492 & $\begin{array}{l}6.49 \mathrm{E}- \\
02\end{array}$ & $\begin{array}{l}5.64344 \\
E-15\end{array}$ \\
\hline $\begin{array}{l}11 / 7 / 20 \\
20\end{array}$ & $\begin{array}{l}0.9656058 \\
88\end{array}$ & $\begin{array}{l}0.0024905 \\
71\end{array}$ & $\begin{array}{l}3.84036 \mathrm{E}- \\
05\end{array}$ & $\begin{array}{l}0.0009682 \\
84\end{array}$ & $\begin{array}{l}0.4263667 \\
18\end{array}$ & $\begin{array}{l}0.0056237 \\
04\end{array}$ & $\begin{array}{l}0.068014 \\
36\end{array}$ & $\begin{array}{l}6.80 \mathrm{E}- \\
02\end{array}$ & $\begin{array}{l}3.43265 \\
E-16\end{array}$ \\
\hline $\begin{array}{l}12 / 7 / 20 \\
20\end{array}$ & $\begin{array}{l}0.9749417 \\
16\end{array}$ & $\begin{array}{l}0.0354176 \\
93\end{array}$ & $\begin{array}{l}7.38167 \mathrm{E}- \\
05\end{array}$ & $\begin{array}{l}7.38167 E- \\
05\end{array}$ & $\begin{array}{l}0.4255033 \\
13\end{array}$ & $\begin{array}{l}0.0014695 \\
57\end{array}$ & $\begin{array}{l}0.071317 \\
28\end{array}$ & $\begin{array}{l}7.13 \mathrm{E}- \\
02\end{array}$ & $\begin{array}{l}2.36257 \\
E-13\end{array}$ \\
\hline $\begin{array}{l}14 / 7 / 20 \\
20\end{array}$ & $\begin{array}{l}0.9684765 \\
83\end{array}$ & $\begin{array}{l}0.0357525 \\
97\end{array}$ & $\begin{array}{l}5.16671 \mathrm{E}- \\
05\end{array}$ & $\begin{array}{l}0.0001047 \\
56\end{array}$ & $\begin{array}{l}0.4091689 \\
67\end{array}$ & $\begin{array}{l}0.0067051 \\
69\end{array}$ & $\begin{array}{l}0.078571 \\
84\end{array}$ & $\begin{array}{l}7.86 \mathrm{E}- \\
02\end{array}$ & $\begin{array}{l}2.14368 \\
E-13\end{array}$ \\
\hline $\begin{array}{l}15 / 7 / 20 \\
20\end{array}$ & $\begin{array}{l}0.9776597 \\
97\end{array}$ & $\begin{array}{l}0.0361726 \\
56\end{array}$ & $\begin{array}{l}7.99106 \mathrm{E}- \\
05\end{array}$ & $\begin{array}{l}7.99106 \mathrm{E}- \\
05\end{array}$ & $\begin{array}{l}0.3957742 \\
18\end{array}$ & $\begin{array}{l}0.0140060 \\
78\end{array}$ & 0.082535 & $\begin{array}{l}8.25 \mathrm{E}- \\
02\end{array}$ & $\begin{array}{l}2.31899 \\
E-13\end{array}$ \\
\hline
\end{tabular}

This publication is licensed under Creative Commons Attribution CC BY. 
International Journal of Scientific and Research Publications, Volume 11, Issue 5, May 2021

408 ISSN $2250-3153$

\begin{tabular}{|l|l|l|l|l|l|l|l|l|}
\hline $18 / 7 / 20$ & 0.9999826 & 0.0383686 & $1.09523 \mathrm{E}-$ & 0.0001384 & 0.3869923 & 0.0021825 & 0.095837 \\
20 & 8 & 87 & 05 & 4 & 48 & $9.58 \mathrm{E}-$ & $\begin{array}{l}1.4633 \mathrm{E} \\
-13\end{array}$ \\
\hline $19 / 7 / 20$ & 0.9410876 & 0.0348809 & $1.00764 \mathrm{E}-$ & 0.0001474 & 0.3740749 & 0.0074293 & 0.100761 & $1.01 \mathrm{E}-$ \\
20 & 68 & 43 & 05 & 18 & 2 & 7 & 24 & 01 \\
\hline
\end{tabular}

